\title{
Stochastic theory of accelerated detectors in a quantum field
}

\author{
Alpan Raval* and B. L. Hu ${ }^{\dagger}$ \\ Department of Physics, University of Maryland, College Park, Maryland 20742 \\ School of Natural Sciences, Institute for Advanced Study, Princeton, New Jersey 08540 \\ and Department of Physics, Hong Kong University of Science and Technology, Clear Water Bay, Kowloon, Hong Kong \\ James Anglin \\ Theoretical Astrophysics, MS B-288, Los Alamos National Laboratory, New Mexico 87545
}

(Received 23 May 1995)

\begin{abstract}
We analyze the statistical mechanical properties of $n$ detectors in arbitrary states of motion interacting with one another via a quantum field. We use the open system concept and the influence functional method to calculate the influence of quantum fields on detectors in motion, and the mutual influence of detectors via fields. We discuss the difference between self and mutual impedance, advanced and retarded noise, and the relations between noise-correlations and dissipation-propagation. The mutual effects of detectors on one another can be studied from the Langevin equations derived from the influence functional, as it contains the back reaction of the field on the system self-consistently. We show the existence of general fluctuation-dissipation relations, and for trajectories without event horizons, correlation-propagation relations, which succinctly encapsulate these quantum statistical phenomena. These findings serve to clarify some existing confusions on the accelerated detector problem. The general methodology presented here could also serve as a platform to explore the quantum statistical properties of particles and fields, with practical applications in atomic and optical physics problems. [S0556-2821(96)03912-4]
\end{abstract}

PACS number(s): 32.80.-t, 04.62.+v, 05.40.+j, 42.50.Lc

\section{INTRODUCTION}

The physics of accelerated detectors became an interesting subject of investigation when Unruh showed that a detector moving with uniform acceleration $a$ sees the vacuum state of some quantum field in Minkowski space as a thermal bath with temperature $T_{U}=\hbar a / 2 \pi c k_{B}$ (where $\hbar, k_{B}, c$ are the Planck and Boltzmann's constants, and the speed of light respectively) [1]. This seminal work which uses the structure of quantum field theory of Rindler space explored by Fulling [2], and the Bogolubov transformation ideas invented by Parker for cosmological particle creation [3] earlier, draws a clear parallel with the fundamental discovery of Hawking radiation in black holes [4]. The discovery of Unruh effect (see also [5]) sets off the first wave of activities on this subject. The state-of-the-art understanding of the physics of this problem in this first stage of work is represented by the paper of Unruh and Wald [6]. We refer the readers to the reviews of Sciama, Candelas, and Deutsch [7], Tagaki [8], and Ginzburg and Frolov [9].

The second stage of investigation on this problem was initiated by the inquiry of Grove [10], who challenged the prevailing view and asked the question whether the detector actually radiates. This was answered in the negative by an inspiring paper of Raine, Sciama, and Grove (RSG) [11] who considered an exactly solvable harmonic oscillator detector model and analyzed what an inertial observer sees in the forward light cone of the accelerating detector via a Langevin equation. Unruh [12] performed an independent calcula-

\footnotetext{
*Electronic address: raval@umdhep.umd.edu

†Electronic address: hu@umdhep.umd.edu

ॠElectronic address: anglin@tdo-serv.lanl.gov
}

tion and concurred with the findings of RSG to the extent that the energy-momentum tensor of the field as modified by the presence of the accelerating detector vanishes over most of the spacetime (except on the horizons). However, he also showed the existence of extra terms in the two-point function of the field beyond its value in the absence of the accelerating detector, and argued that these terms would contribute to the excitation of a detector placed in the forward light cone. These terms were missed out in RSG. Following these exchanges, there was a recent renewed interest in this problem, notably the series of papers by Massar, Parentani, and Brout [13] (MPB), who gave a detailed analysis via Hamiltonian quantum mechanics of the two-point function and pointed out that the missing terms contribute to a polarization cloud around the accelerating detector; Hinterleitner [14], who independently discussed the back reaction of the detector on the field using a slightly different yet exactly solvable model and arrived at conclusions similar to MPB; and Audretsch and Müller [15], who explored nonlocal pair correlations in accelerated detectors. However, the physical significance of the polarization cloud, its connection to the noise experienced by another detector, and to the inherent correlations in the free Minkowski vacuum, remain largely unexplored.

Beginning with this work we would like to add a new dimension to this problem and open up the third stage of investigation. The new emphasis is in exploring the statistical mechanics of particles and fields, and in particular, moving detectors on arbitrary trajectories. We analyze the stochastic properties of quantum fields and discuss this problem in terms of quantum noise, correlation, and dissipation. We use the open system concept and the influence functional method [30] to treat a system of $n$ detectors interacting with a scalar field. This method enables one to examine the influence of detectors in motion on quantum fields, the mutual influences of detectors via fields, as well as the back reaction 
of fields on detectors in a self-consistent manner.

As explained earlier [16], the influence functional method is a generalization of the powerful effective action method in quantum field theory for treating back reaction problems, which also incorporates statistical mechanics notions such as noise, fluctuations, decoherence, and dissipation. Indeed, one of us has long held the viewpoint $[17,18]$ that to get a more profound understanding of the meaning of Unruh and Hawking effects and the black hole information and back reaction problems one cannot be satisfied with the equilibrium thermodynamics description. It is necessary to probe deeper into the statistical properties of quantum fields, their correlations and dynamics, coherence and decoherence of the particlefield system, the relation of quantum noise and thermal radiance, fluctuation-dissipation relations, etc. Earlier investigation of correlation and dissipation in the BoltzmannBogoliubov-Born-Green-Kirkwood-Yvon (BBGKY) scheme [19-21] and the properties of noise and fluctuations in the Langevin framework [22-24] are essential preparations for tackling such problems at a deeper level. ${ }^{1}$

With this theoretical perspective in mind, we have recently begun a systematic study of the accelerated detector problem $[25,23]$. We show that thermal radiance can be understood as originating from quantum noise under different kinematical (moving detector) and dynamical (cosmology) excitations. The aim of this paper is to (1) show on both the conceptual and technical levels the power and versatility of this new method, (2) settle some open questions and clarify some existing confusions, such as the existence of radiation and polarization, solely from an analysis of detector response, (3) introduce new concepts such as self and mutual impedance, advanced and retarded noise, fluctuationdissipation and correlation-propagation relations using the accelerated detectors problem as an example, and finally, (4) suggest new avenues of investigations into the statistical mechanics of particle and fields, including black hole physics.

Employing a set of coupled stochastic equations for the detector dynamics, we analyze the influence of an accelerated detector on a probe which is not allowed to causally influence the accelerated detector itself. We find, as did [1113], that most of the terms in the correlations of the stochastic force acting on the probe cancel each other. This cancellation is understood in the light of a correlation-propagation relation, derived as a simple construction from the fluctuation-dissipation relation for the accelerated detector. Such a relation can be equivalently viewed as a construction of the free field two-point function for each point on either trajectory from the two-point function along the uniformly accelerated trajectory alone. The remaining terms, which contribute to the excitation of the probe, are shown to represent correlations of the free field across the future horizon of the accelerating detector. In this problem, the dissipative properties of either detector remain unchanged by the presence of the other. This happens because the probe cannot influence the accelerated detector. However, the stochastic force acting on the probe plays a nontrivial role.

We also consider the problem of two inertial detectors which can back react on each other. This mutual back reac-

\footnotetext{
${ }^{1}$ Our view is thus most akin to that espoused by Sciama [26].
}

tion changes the self-impedance functions of these detectors, and introduces mutual impedances as well. The dissipative properties of each detector are thus altered because of the presence of the other one. This physical effect is in a sense complementary to the effects manifested in the accelerated detector problem, where the probe does not back react on the accelerated detector.

The paper is organized as follows. In Sec. II we develop the influence functional formalism describing the influence of a massless scalar field on a system of an arbitrary number of detectors moving on arbitrary trajectories. The field modes are integrated out in this formalism, and effective stochastic equations of motion for the various detectors are obtained. In Sec. III we consider some applications of this formalism to three simple cases, the primary one being the analysis of two inertial detectors coupled to the same quantum field. In Sec. IV we treat the RSG excitation of a probe in the presence of a uniformly accelerating detector. In Sec. V we show the existence of fluctuation-dissipation relations governing the detector system. These relations are used as a starting point for obtaining more general relations between the correlations of various detectors and the radiation mediated by them. Such relations are also discussed in the specific context of the RSG model. Finally, in Appendices A and B, we point out problems associated with the uncorrelated detector-field initial state in a minimally coupled model, and argue that these problems are removed in a derivative coupling model. We present a simple prescription for switching from one model to the other.

\section{SCALAR ELECTRODYNAMICS OR MINIMAL COUPLING MODEL}

The paper by Raine, Sciama, and Grove uses the scalar electrodynamic or "minimal" coupling of oscillators to a scalar field in $1+1$ dimensions. This coupling provides a positive definite Hamiltonian, and is of interest because it resembles the actual coupling of charged particles to an electromagnetic field. In this section, we derive the influence functional describing the effect of a scalar field on the dynamics of an arbitrary number of detectors modelled as minimally coupled oscillators. The detectors move along arbitrary trajectories. We assume that the field and the system of detectors are initially decoupled from each other, and that the field is initially in the Minkowski vacuum state. The formalism can be simply extended to higher dimensions, and to different choices of initial state for the field. We also obtain coupled Langevin equations for the detector system.

\section{A. Influence functional for $N$ arbitrarily moving detectors}

Consider $N$ detectors $i=1, \ldots, N$ in $1+1$ dimensions with internal oscillator coordinates $Q_{i}\left(\tau_{i}\right)$, and trajectories $\left(x_{i}\left(\tau_{i}\right), t_{i}\left(\tau_{i}\right)\right), \tau_{i}$ being a parameter along the trajectory of detector $i$. In the following analysis, we do not need to assume that $\tau_{i}$ is the proper time, although this is, in most cases, a convenient choice. However, we will assume hereafter that the trajectories $\left(t_{i}\left(\tau_{i}\right), x_{i}\left(\tau_{i}\right)\right)$ are smooth and that the parameters $\tau_{i}$ are chosen such that $t_{i}\left(\tau_{i}\right)$ is a strictly increasing function of $\tau_{i}$. 
The detectors are coupled to a massless scalar field $\phi(x, t)$ via the interaction action

$S_{\mathrm{int}}=\sum_{i} e_{i} \int_{-\infty}^{t_{i}^{-1}(T)} d \tau_{i} s_{i}\left(\tau_{i}\right) \frac{d Q_{i}}{d \tau_{i}} \phi\left(x_{i}\left(\tau_{i}\right), t_{i}\left(\tau_{i}\right)\right)$.

Here, $T$ is a global Minkowski time coordinate which defines a spacelike hypersurface, $e_{i}$ denotes the coupling constant of detector $i$ to the field, $s_{i}\left(\tau_{i}\right)$ is the switching function for detector $i$ (typically a step function), and $t_{i}^{-1}$ is the inverse function of $t_{i} \cdot t_{i}^{-1}(T)$ is, therefore, the value of $\tau_{i}$ at the point of intersection of the spacelike hypersurface defined by $T$ with the trajectory of detector $i$. Note that the strictly increasing property of $t_{i}\left(\tau_{i}\right)$ implies that the inverse, if it exists, is unique.

The action of the system of detectors is

$$
S_{\mathrm{osc}}=\frac{1}{2} \sum_{i} \int_{-\infty}^{t_{i}^{-1}(T)} d \tau_{i}\left[\left(\partial_{\tau_{i}} Q_{i}\right)^{2}-\Omega_{i}^{2} Q_{i}^{2}\right] .
$$

The scalar field action is given by

$$
S_{\text {field }}=\frac{1}{2} \int_{-\infty}^{T} d t \int d x\left[\left(\partial_{t} \phi\right)^{2}-\left(\partial_{x} \phi\right)^{2}\right]
$$

and the complete action

$$
S=S_{\text {field }}+S_{\text {osc }}+S_{\text {int }} .
$$

Expanding the field in normal modes,

$$
\phi(x, t)=\sqrt{\frac{2}{L}} \sum_{k}{ }^{\prime}\left[q_{k}^{+}(t) \cos k x+q_{k}^{-}(t) \sin k x\right],
$$

where $\Sigma_{k}^{\prime}$ denotes that the summation is restricted to the upper half $k$ space, $k>0$. Then the action for the scalar field is given by $(\sigma=+,-)$

$$
S_{\text {field }}=\frac{1}{2} \sum_{k, \sigma}{ }^{\prime}\left[\left(\dot{q}_{k}^{\sigma}\right)^{2}-\omega_{k}^{2} q_{k}^{2}\right]
$$

and the interaction action is

$$
\begin{aligned}
& S_{\mathrm{int}}=\sum_{i} e_{i} \sqrt{\frac{2}{L}} \int_{-\infty}^{t_{i}^{-1}(T)} d \tau_{i} \frac{d Q_{i}}{d \tau_{i}} \sum_{k}{ }^{\prime}\left[q_{k}^{+}\left(t_{i}\left(\tau_{i}\right)\right) \cos k x_{i}\left(\tau_{i}\right)\right. \\
& \left.+q_{k}^{-}\left(t_{i}\left(\tau_{i}\right)\right) \sin k x_{i}\left(\tau_{i}\right)\right] s_{i}\left(\tau_{i}\right) \\
& =\sum_{i} e_{i} \sqrt{\frac{2}{L}} \int_{-\infty}^{\infty} d t \int_{-\infty}^{t_{i}^{-1}(T)} d \tau_{i} \delta\left(t-t_{i}\left(\tau_{i}\right)\right) \frac{d Q_{i}}{d \tau_{i}} \\
& \times \sum_{k}{ }^{\prime}\left[q_{k}^{+}(t) \cos k x_{i}\left(\tau_{i}\right)+q_{k}^{-}(t) \sin k x_{i}\left(\tau_{i}\right)\right] s_{i}\left(\tau_{i}\right) .
\end{aligned}
$$

We have $t_{i}\left(\tau_{i}\right)<T$, which follows from $\tau_{i}<t_{i}^{-1}(T)$ and the property that $t_{i}\left(\tau_{i}\right)$ is a strictly increasing function. Hence, we may replace the upper limit of the $d t$ integration by $T$. This replacement leads to the expression

$$
S_{\mathrm{int}}=-\sum_{k, \sigma}^{\prime} \int_{-\infty}^{T} d t J_{k}^{\sigma}(t) q_{k}^{\sigma}(t)
$$

where

$$
J_{k}^{\sigma}(t)=-\sum_{i} e_{i} \sqrt{\frac{2}{L}} \int_{-\infty}^{t_{i}^{-1}(T)} d \tau_{i} \delta\left(t-t_{i}\left(\tau_{i}\right)\right) \frac{d Q_{i}}{d \tau_{i}} u_{k}^{\sigma}\left(\tau_{i}\right) s_{i}\left(\tau_{i}\right)
$$

and

$$
u_{k}^{+}\left(\tau_{i}\right)=\cos k x_{i}\left(\tau_{i}\right), \quad u_{k}^{-}\left(\tau_{i}\right)=\sin k x_{i}\left(\tau_{i}\right) .
$$

The action $S_{\text {field }}+S_{\text {int }}$, therefore, describes a system of decoupled harmonic oscillators each driven by separate source terms. The zero temperature influence functional (corresponding to the initial state of the field being the Minkowski vacuum) for this system has the form [22]

$$
\begin{aligned}
\mathcal{F}\left[J, J^{\prime}\right]= & \exp \left\{-\frac{1}{\hbar} \sum_{k, \sigma}^{\prime} \int_{-\infty}^{T} d s \int_{-\infty}^{s} d s^{\prime}\left[J_{k}^{\sigma}(s)-J_{k}^{\prime \sigma}(s)\right]\right. \\
& \left.\times\left[\zeta_{k}\left(s, s^{\prime}\right) J_{k}^{\sigma}\left(s^{\prime}\right)-\zeta_{k}^{*}\left(s, s^{\prime}\right) J_{k}^{\prime \sigma}\left(s^{\prime}\right)\right]\right\}, \quad(2.11)
\end{aligned}
$$

where

$$
\zeta_{k} \equiv \nu_{k}+i \mu_{k}=\frac{1}{2 \omega_{k}} \exp \left[-i \omega_{k}\left(s-s^{\prime}\right)\right]
$$

If the field is initially in a thermal state, the influence functional has the same form as above, and the quantity $\zeta_{k}$ becomes

$$
\zeta_{k}=\frac{1}{2 \omega_{k}}\left[\operatorname{coth}\left(\frac{\beta \omega_{k} \hbar}{2}\right) \cos \omega_{k}\left(s-s^{\prime}\right)-i \sin \omega_{k}\left(s-s^{\prime}\right)\right],
$$

$\beta$ being the inverse temperature. We shall restrict our attention to the zero temperature case.

Substituting for the $J_{k}^{\sigma}$, s in the influence functional, and carrying out the $\delta$-function integrations, one obtains

$$
\begin{aligned}
\mathcal{F}\left[\{Q\} ;\left\{Q^{\prime}\right\}\right]= & \exp \left\{-\frac{1}{\hbar}\left[\sum_{i, j=1}^{N} \int_{-\infty}^{t_{i}^{-1}(T)}\right.\right. \\
& \times d \tau_{i} s_{i}\left(\tau_{i}\right) \int_{-\infty}^{t_{j}^{-1}\left(t_{i}\left(\tau_{i}\right)\right)} d \tau_{j}^{\prime} s_{j}\left(\tau_{j}^{\prime}\right) \\
& \times\left(\frac{d Q_{i}}{d \tau_{i}}-\frac{d Q_{i}^{\prime}}{d \tau_{i}}\right)\left(Z_{i j}\left(\tau_{i}, \tau_{j}^{\prime}\right) \frac{d Q_{j}}{d \tau_{j}^{\prime}}\right. \\
& \left.\left.\left.-Z_{i j}^{*}\left(\tau_{i}, \tau_{j}^{\prime}\right) \frac{d Q_{j}^{\prime}}{d \tau_{j}^{\prime}}\right)\right]\right\},
\end{aligned}
$$

where

$$
Z_{i j}\left(\tau_{i}, \tau_{j}^{\prime}\right)=\frac{2}{L} e_{i} e_{j} \sum_{k, \sigma}^{\prime} \zeta_{k}\left(t_{i}\left(\tau_{i}\right), t_{j}\left(\tau_{j}^{\prime}\right)\right) u_{k}^{\sigma}\left(\tau_{i}\right) u_{k}^{\sigma}\left(\tau_{j}^{\prime}\right)
$$


In the above, the continuum limit in the mode sum is recovered through the replacement $\Sigma_{k}^{\prime} \rightarrow(L / 2 \pi) \int_{0}^{\infty} d k$. We then obtain, after substituting for $u_{k}^{\sigma}$ and $\zeta_{k}$,

$$
\begin{aligned}
Z_{i j}\left(\tau_{i}, \tau_{j}^{\prime}\right)= & \frac{e_{i} e_{j}}{2 \pi} \int_{0}^{\infty} \frac{d k}{k} \exp \left\{-i k\left[t_{i}\left(\tau_{i}\right)\right.\right. \\
& \left.\left.-t_{j}\left(\tau_{j}^{\prime}\right)\right]\right\} \cos k\left(x_{i}\left(\tau_{i}\right)-x_{j}\left(\tau_{j}^{\prime}\right)\right) .
\end{aligned}
$$

In this form, $Z_{i j}$ is proportional to the two-point function of the free scalar field in the Minkowski vacuum, evaluated for the two points lying on trajectories $i$ and $j$ of the detector system. It obeys the symmetry relation

$$
Z_{i j}\left(\tau_{i}, \tau_{j}^{\prime}\right)=Z_{j i}^{*}\left(\tau_{j}^{\prime}, \tau_{i}\right) .
$$

Corresponding to (2.12), we may also split $Z_{i j}$ into its real and imaginary parts. Thus, we define

$$
Z_{i j}\left(\tau_{i}, \tau_{j}^{\prime}\right)=\widetilde{\nu}_{i j}\left(\tau_{i}, \tau_{j}^{\prime}\right)+i \widetilde{\mu}_{i j}\left(\tau_{i}, \tau_{j}^{\prime}\right)
$$

where

$$
\begin{aligned}
\widetilde{\nu}_{i j}\left(\tau_{i}, \tau_{j}^{\prime}\right)= & \frac{e_{i} e_{j}}{2 \pi} \int_{0}^{\infty} \frac{d k}{k} \cos k\left(t_{i}\left(\tau_{i}\right)-t_{j}\left(\tau_{j}^{\prime}\right)\right) \\
& \times \cos k\left(x_{i}\left(\tau_{i}\right)-x_{j}\left(\tau_{j}^{\prime}\right)\right), \\
\tilde{\mu}_{i j}\left(\tau_{i}, \tau_{j}^{\prime}\right)= & -\frac{e_{i} e_{j}}{2 \pi} \int_{0}^{\infty} \frac{d k}{k} \sin k\left(t_{i}\left(\tau_{i}\right)-t_{j}\left(\tau_{j}^{\prime}\right)\right) \\
& \times \cos k\left(x_{i}\left(\tau_{i}\right)-x_{j}\left(\tau_{j}^{\prime}\right)\right) .
\end{aligned}
$$

$\widetilde{\nu}$ and $\tilde{\mu}$ are proportional to the anticommutator and the commutator of the field in the Minkowski vacuum, respectively.

The quantities $Z_{i j}$ are also conveniently expressed in terms of advanced and retarded null coordinates $v_{i}\left(\tau_{i}\right)=t_{i}\left(\tau_{i}\right)+x_{i}\left(\tau_{i}\right)$ and $u_{i}\left(\tau_{i}\right)=t_{i}\left(\tau_{i}\right)-x_{i}\left(\tau_{i}\right)$, as

$$
Z_{i j}\left(\tau_{i}, \tau_{j}^{\prime}\right)=Z_{i j}^{a}\left(\tau_{i}, \tau_{j}^{\prime}\right)+Z_{i j}^{r}\left(\tau_{i}, \tau_{j}^{\prime}\right)
$$

where

$$
\begin{aligned}
& Z_{i j}^{a}\left(\tau_{i}, \tau_{j}^{\prime}\right)=\frac{e_{i} e_{j}}{4 \pi} \int_{0}^{\infty} \frac{d k}{k} \exp \left[-i k\left(v_{i}\left(\tau_{i}\right)-v_{j}\left(\tau_{j}^{\prime}\right)\right)\right], \\
& Z_{i j}^{r}\left(\tau_{i}, \tau_{j}^{\prime}\right)=\frac{e_{i} e_{j}}{4 \pi} \int_{0}^{\infty} \frac{d k}{k} \exp \left[-i k\left(u_{i}\left(\tau_{i}\right)-u_{j}\left(\tau_{j}^{\prime}\right)\right)\right],
\end{aligned}
$$

and the superscripts $a$ and $r$ denote advanced and retarded, respectively. ${ }^{2}$ Similar decompositions for $\tilde{\nu}_{i j}$ and $\tilde{\mu}_{i j}$ thus follow.

\footnotetext{
2The terminology "advanced" and "retarded" refers to the null coordinates. Equivalently, they can be called "left moving" and "right moving,"' respectively, when the sense of motion refers to the future direction in time. This terminology is used in wave and string theories.
}

The influence functional, along with the action for the detector system, can be employed to obtain the propagator for the density matrix of the system of detectors. This propagator will contain complete information about the dynamics of the detectors. However, we shall take the alternative approach of deriving Langevin equations for the detector system in order to describe its dynamics.

\section{B. Langevin equations}

In this subsection, we wish to derive the effective stochastic equations of motion for the $N$-detector system. In the previous subsection, we integrated out the field degrees of freedom. The effect of this is to introduce long-range interactions between the various detectors.

Going back to the form (2.11) for the influence functional, we define the center of mass and relative variables;

$$
\begin{gathered}
J_{k}^{+\sigma}(s)=\left(J_{k}^{\sigma}(s)+J_{k}^{\prime \sigma}(s)\right) / 2, \\
J_{k}^{-\sigma}(s)=J_{k}^{\sigma}(s)-J_{k}^{\prime \sigma}(s) .
\end{gathered}
$$

Correspondingly, we also find it convenient to define

$$
\begin{gathered}
Q_{i}^{+}\left(\tau_{i}\right)=\left(Q_{i}\left(\tau_{i}\right)+Q_{i}^{\prime}\left(\tau_{i}\right)\right) / 2, \\
Q_{i}^{-}\left(\tau_{i}\right)=Q_{i}\left(\tau_{i}\right)-Q_{i}^{\prime}\left(\tau_{i}\right) .
\end{gathered}
$$

Then, Eq. (2.11) yields

$$
\begin{gathered}
\left|\mathcal{F}\left[J, J^{\prime}\right]\right|=\exp \left\{-\frac{1}{\hbar} \sum_{k, \sigma} \int_{-\infty}^{T} d s \int_{-\infty}^{s} d s^{\prime} J_{k}^{-\sigma}(s)\right. \\
\left.\times \nu_{k}\left(s, s^{\prime}\right) J_{k}^{-\sigma}\left(s^{\prime}\right)\right\} \\
=\int \Pi_{k, \sigma}^{\prime}\left(\mathcal{D} \xi_{k}^{\sigma} P\left[\xi_{k}^{\sigma}\right]\right) \exp \left(-\frac{i}{\hbar} \sum_{k, \sigma}^{\prime} \int_{-\infty}^{T} d s J_{k}^{-\sigma}(s) \xi_{k}^{\sigma}(s)\right) .
\end{gathered}
$$

$|\mathcal{F}|$ is the absolute value of $\mathcal{F}$, containing the kernel $\nu_{k}$. The phase of $\mathcal{F}$ contains the kernel $\mu_{k}$. In the second equality, we have used a functional Gaussian integral identity, $P\left[\xi_{k}^{\sigma}\right]$ being the positive definite measure

$$
\begin{aligned}
P\left[\xi_{k}^{\sigma}\right]= & N \exp \\
& \times\left\{-\frac{1}{2 \hbar} \int_{-\infty}^{T} d s \int_{-\infty}^{T} d s^{\prime} \xi_{k}^{\sigma}(s) \nu_{k}^{-1}\left(s, s^{\prime}\right) \xi_{k}^{\sigma}\left(s^{\prime}\right)\right\},
\end{aligned}
$$

normalized to unity. It can, therefore, be interpreted as a probability distribution over the function space $\xi_{k}^{\sigma}$.

The influence functional can thus be expressed as 


$$
\begin{aligned}
\left.\mathcal{F}\{Q\},\left\{Q^{\prime}\right\}\right]= & \exp \left\{-\frac{i}{\hbar} \sum_{k, \sigma}^{\prime} \int_{-\infty}^{T} d s J_{k}^{-\sigma}(s)\left[\xi_{k}^{\sigma}(s)\right.\right. \\
& \left.\left.\left.+2 \int_{-\infty}^{s} d s^{\prime} \mu_{k}\left(s, s^{\prime}\right) J_{k}^{+\sigma}\left(s^{\prime}\right)\right]\right\}\right\rangle \\
\equiv & \left\langle\exp \left(\frac{i}{\hbar} S_{\text {inf }}\right)\right\rangle
\end{aligned}
$$

where angular brackets denote expectation value with respect to the joint distribution $\Pi_{k, \sigma}^{\prime} P\left[\xi_{k}^{\sigma}\right] . S_{\text {inf }}$ will be called the stochastic influence action. We find

$$
\begin{gathered}
\left\langle\xi_{k}^{\sigma}(s)\right\rangle=0, \\
\left\langle\left\{\xi_{k}^{\sigma}(s), \xi_{k^{\prime}}^{\sigma^{\prime}}\left(s^{\prime}\right)\right\}\right\rangle=\hbar \delta_{k k^{\prime}} \delta_{\sigma \sigma^{\prime}} \nu_{k}\left(s, s^{\prime}\right),
\end{gathered}
$$

where $\{$,$\} denotes the anticommutator.$

Substituting for $J_{k}^{-\sigma}$ and $J_{k}^{+\sigma}$ in terms of the detector degrees of freedom $\left\{Q_{i}\right\}$, the stochastic influence action $S_{\text {inf }}$ is obtained as

$$
\begin{aligned}
S_{\mathrm{inf}}= & -\sum_{i=1}^{N} \int_{-\infty}^{t_{i}^{-1}(T)} d \tau_{i} \frac{d Q_{i}^{-}}{d \tau_{i}} s_{i}\left(\tau_{i}\right)\left[\eta_{i}\left(\tau_{i}\right)\right. \\
& \left.+2 \sum_{j=1}^{N} \int_{-\infty}^{-1\left(t_{i}\left(\tau_{i}\right)\right)} d \tau_{j}^{\prime} \frac{d Q_{j}^{+}}{d \tau_{j}^{\prime}} s_{j}\left(\tau_{j}^{\prime}\right) \tilde{\mu}_{i j}\left(\tau_{i}, \tau_{j}^{\prime}\right)\right]
\end{aligned}
$$

with

$$
\eta_{i}\left(\tau_{i}\right)=e_{i} \sum_{k, \sigma}^{\prime} \sqrt{\frac{2}{L}} u_{k}^{\sigma}\left(\tau_{i}\right) \xi_{k}^{\sigma}\left(t_{i}\left(\tau_{i}\right)\right)
$$

From Eq. (2.29) we see that the quantities $\tilde{\mu}_{i j}, i \neq j$ mediate long-range interactions between the various detectors and the quantities $\tilde{\mu}_{i i}$ describe self-interaction of each detector because of its interaction with the field. This self-interaction typically manifests itself as a dissipative (or radiation reaction) force in the dynamics of the detectors. We will, therefore, refer to $\tilde{\mu}_{i j}, i \neq j$ as a "propagation kernel," and $\tilde{\mu}_{i i}$ as a "dissipation kernel."

We now turn to the interpretation of the quantities $\eta_{i}$. They appear as source terms in the effective action of the detector system. Also, being linear combinations of the quantities $\xi_{k}^{\sigma}$, they are stochastic in nature. Indeed, from Eqs. (2.28) and (2.30), we can obtain

$$
\begin{aligned}
&\left\langle\eta_{i}\left(\tau_{i}\right)\right\rangle=0 \\
&\left\langle\left\{\eta_{i}\left(\tau_{i}\right), \eta_{j}\left(\tau_{j}^{\prime}\right)\right\}\right\rangle= e_{i} e_{j} \sum_{k, \sigma}^{\prime} \sum_{k^{\prime}, \sigma^{\prime}}^{\prime} u_{k}^{\sigma}\left(\tau_{i}\right) u_{k^{\prime}}^{\sigma^{\prime}}\left(\tau_{j}^{\prime}\right)\left(\frac{2}{L}\right) \\
& \times\left\langle\xi_{k}^{\sigma}\left(t_{i}\left(\tau_{i}\right)\right) \xi_{k^{\prime}}^{\sigma^{\prime}}\left(t_{j}\left(\tau_{j}^{\prime}\right)\right)\right\rangle \\
&= \hbar \widetilde{\nu}_{i j}\left(\tau_{i}, \tau_{j}^{\prime}\right) .
\end{aligned}
$$

Thus, $\widetilde{\nu}_{i j}$ appears as a correlator of the stochastic forces $\eta_{i}$ and $\eta_{j}$. Along a fixed trajectory, this correlation manifests as noise in the detector dynamics. Hence, we call $\widetilde{\nu}_{i i}$ a "'noise kernel'" and $\widetilde{\nu}_{i j}, i \neq j$, a "correlation kernel.,,3

The full stochastic effective action for the $N$-detector system is given by

$$
S_{\text {eff }}=S_{\text {osc }}+S_{\text {inf }}
$$

We may now express this in terms of the variables $Q_{i}^{+}$and $Q_{i}^{-}$defined earlier. Thus, we obtain

$$
\begin{aligned}
S_{\mathrm{eff}}= & \sum_{i=1}^{N} \int_{-\infty}^{t_{i}^{-1}(T)} d \tau_{i}\left[\dot{Q}_{i}^{-} \dot{Q}_{i}^{+}-\Omega_{i}^{2} Q_{i}^{-} Q_{i}^{+}-\dot{Q}_{i}^{-} s_{i}\left(\tau_{i}\right) \eta_{i}\left(\tau_{i}\right)\right. \\
& \left.-2 \dot{Q}_{i}^{-} s_{i}\left(\tau_{i}\right) \sum_{j=1}^{N} \int_{-\infty}^{-1\left(t_{i}\left(\tau_{i}\right)\right)} d \tau_{j}^{\prime} \dot{Q}_{j^{\prime}}^{+} s_{j}\left(\tau_{j}^{\prime}\right) \tilde{\mu}_{i j}\left(\tau_{i}, \tau_{j}^{\prime}\right)\right],
\end{aligned}
$$

where $\dot{f}_{i} \equiv d f_{i} / d \tau_{i}, \dot{f}_{j^{\prime}} \equiv d f_{j} / d \tau_{j}^{\prime}$.

Extremizing the effective action with respect to $Q_{i}^{-}$and setting $Q_{i}=Q_{i}^{\prime}$ at the end [22], we obtain a set of coupled equations of motion, the Langevin equations, for the system of detectors:

$$
\begin{aligned}
& \frac{d^{2} Q_{i}}{d \tau_{i}^{2}}-2 \sum_{j=1}^{N} \int_{-\infty}^{-1\left(t_{i}\left(\tau_{i}\right)\right)} d \tau_{j}^{\prime} s_{j}\left(\tau_{j}^{\prime}\right) \frac{d}{d \tau_{i}}\left(s_{i}\left(\tau_{i}\right) \tilde{\mu}_{i j}\left(\tau_{i}, \tau_{j}^{\prime}\right)\right) \frac{d Q_{j}}{d \tau_{j}^{\prime}} \\
& \quad+\Omega_{i}^{2} Q_{i}=\frac{d}{d \tau_{i}}\left(s_{i}\left(\tau_{i}\right) \eta_{i}\left(\tau_{i}\right)\right) .
\end{aligned}
$$

Because of the back reaction of each detector on the field, and consequently on other detectors, the effective dynamics of the detector system is highly nontrivial and, as such, can be solved in closed form only for simple trajectories or under simplifying assumptions such as ignoring the back reaction of certain detectors on the field. For instance, if we choose to ignore the back reaction of detector $i$ on the field, this can be effected by setting $\widetilde{\mu}_{j i}=0$, for all $j$, including $j=i$, while at the same time keeping $\widetilde{\mu}_{i j} \neq 0$ for $j \neq i$. The particular case $\widetilde{\mu}_{i i}=0$ amounts to ignoring the radiation reaction of detector $i$. This is necessary because the radiation reaction effect arises because of a modification of the field in the vicinity of the detector as a consequence of the back reaction of the detector on the field.

Of course, it is, in general, inconsistent to ignore the back reaction of a detector, as it leads to a direct violation of the symmetry (2.17). As is well known, it also leads to unphysical predictions. For example, in the treatment of an atom on an inertial trajectory, coupled to a quantum field, balance of vacuum fluctuations and radiation reaction is necessary to ensure the stability of the ground state. As explained above, ignoring back reaction implies ignoring the radiation reaction force. Such a treatment would render the ground state unstable.

\footnotetext{
${ }^{3}$ The distinction between noise and correlation is unnecessary from the point of view of the field. "Noise," as used here, also represents free field correlations for points on a single trajectory. However, from the point of view of each detector, these two quantities play a different role. Hence, the choice of terminology.
} 
However, in certain cases, the quantities $\tilde{\mu}_{j i}$ may not contribute to the dynamics of detector $j$, as in Sec. IV below, where the trajectory of one detector is always outside the causal future of the other one. Hence, there is no retarded effect of one of the detectors on the other.

Our formal treatment of the detector-field system is exact in that it includes the full back reaction of the detectors on the field, which is manifested in the coupled Langevin equations of the various detectors. The coupled equations of motion give rise to a sort of "dynamical correlation" between the various detectors. Nondynamical correlations also occur because of the intrinsic correlations in the state of the field (Minkowski vacuum). These correlations are purely quantum mechanical in origin, and they are reflected in the correlators of the stochastic forces, $\widetilde{\nu}_{i j}$. Correlations between stochastic forces on different detectors induce correlations between the coordinates $Q_{i}$ of different detectors.

As we shall show in a later section, our exact treatment makes it possible to demonstrate the existence of generalized fluctuation-dissipation and correlation-propagation relations governing the detector system.

\section{EXAMPLES}

In this and the following section, we consider some applications of the Langevin equations derived in the previous section to the cases of a single detector in the Minkowski vacuum moving on an inertial trajectory, a single detector on a uniformly accelerated trajectory, two detectors on inertial trajectories, and the case of one detector on a uniformly accelerated trajectory and another one on an arbitrary trajectory, functioning as a probe.

The first two examples serve to illustrate the formalism, and describe the well-known physical effects of the dressing of a particle by the field and the thermal Unruh noise experienced by a uniformly accelerated particle.

In the example of two inertial detectors, we introduce the notions of "self" and "mutual" impedances which govern the response of either detector. The effect of the back reaction of each detector on the field and consequently, on the other detector is to introduce the so-called mutual impedance in the detector response as well as to modify the selfimpedance of each detector from its value in the absence of the other one.

In the next section we shall consider the example of one detector on a uniformly accelerated trajectory and a probe, which moves along an unspecified trajectory. We switch on the probe after it intersects the future horizon of the uniformly accelerated detector, so that it cannot causally influence the uniformly accelerated one. Thus, the uniformly accelerated detector in this case is effectively in an unperturbed Unruh heat bath, and this situation mimics most closely the RSG model. The missing terms in the RSG analysis, which contribute to a polarization cloud around the accelerated oscillator, but not to the energy-momentum tensor, lead to a modified noise kernel in the Langevin equation for the probe.

In all cases, we can solve exactly for the detector coordinates, at least in the late time limit (this limit is actually realized at any finite time when the two detectors have been switched on forever, and corresponds to the neglect of transients in the solutions for the detector coordinates).

\section{A. One inertial detector}

Consider the case of one detector moving on an inertial trajectory $x(\tau)=0, t(\tau)=\tau$, and switched on forever $[s(\tau)=1]$. The noise and dissipation kernels take the form

$$
\begin{gathered}
\widetilde{\nu}\left(\tau, \tau^{\prime}\right)=\frac{e^{2}}{2 \pi} \int_{0}^{\infty} \frac{d k}{k} \cos k\left(\tau-\tau^{\prime}\right), \\
\widetilde{\mu}\left(\tau, \tau^{\prime}\right)=-\frac{e^{2}}{2 \pi} \int_{0}^{\infty} \frac{d k}{k} \sin k\left(\tau-\tau^{\prime}\right) .
\end{gathered}
$$

The Langevin equation becomes

$$
\frac{d^{2} Q}{d \tau^{2}}+\frac{e^{2}}{2} \frac{d Q}{d \tau}+\Omega_{0}^{2} Q=\frac{d \eta}{d \tau}
$$

with

$$
\left\langle\left\{\eta(\tau), \eta\left(\tau^{\prime}\right)\right\}\right\rangle=\hbar \widetilde{\nu}\left(\tau, \tau^{\prime}\right) .
$$

It will be convenient to define the dissipation constant $\gamma=e^{2} / 4$. We will restrict our attention to the underdamped case $\left(\gamma<\Omega_{0}\right)$. Note that the form of the dissipation term in Eq. (3.3) above is usually known to arise from the WignerWeisskopf approximation (see, for example, [11]). In this context, however, it turns out to be exact when the continuum limit is taken in the mode sum defining $\tilde{\mu}$.

Introducing the Fourier transform

$$
\begin{gathered}
Q(\tau)=\int_{-\infty}^{\infty} d \omega e^{i \omega \tau} \widetilde{Q}(\omega), \\
\widetilde{Q}(\omega)=\frac{1}{2 \pi} \int_{-\infty}^{\infty} d \omega e^{-i \omega \tau} Q(\tau),
\end{gathered}
$$

and similarly for $\eta(\tau)$, we obtain

$$
\widetilde{Q}(\omega)=\chi_{\omega} \widetilde{\eta}(\omega)
$$

with the impedance function $\chi_{\omega}$ defined as

$$
\chi_{\omega}=i \omega\left(-\omega^{2}+\Omega_{0}^{2}+2 i \omega \gamma\right)^{-1} .
$$

In the above solution for the detector coordinate in frequency space, it should be noted that transients have already been neglected. Transient terms correspond to delta functions in frequency space, the coefficients of these delta functions being determined by the initial conditions. For the complete solution these terms should be added to the right-hand side of Eq. (3.6). We may thus obtain

$$
\left\langle\left\{\widetilde{Q}(\omega), \widetilde{Q}\left(\omega^{\prime}\right)\right\}\right\rangle=\chi_{\omega} \chi_{\omega^{\prime}}\left\langle\left\{\widetilde{\eta}(\omega), \widetilde{\eta}\left(\omega^{\prime}\right)\right\}\right\rangle,
$$

where 


$$
\begin{aligned}
\left\langle\left\{\widetilde{\eta}(\omega), \widetilde{\eta}\left(\omega^{\prime}\right)\right\}\right\rangle= & \frac{1}{4 \pi^{2}} \int_{-\infty}^{\infty} d \tau \int_{-\infty}^{\infty} d \tau^{\prime} e^{-i \omega \tau} \\
& \times e^{-i \omega^{\prime} \tau^{\prime}}\left\langle\left\{\eta(\tau), \eta\left(\tau^{\prime}\right)\right\}\right\rangle \\
= & \hbar \frac{\gamma}{\pi \omega} \delta\left(\omega+\omega^{\prime}\right)[\theta(\omega)-\theta(-\omega)] .
\end{aligned}
$$

We can therefore obtain the correlator of $Q(\tau)$ and $Q\left(\tau^{\prime}\right)$, as

$$
\begin{aligned}
\left\langle\left\{Q(\tau), Q\left(\tau^{\prime}\right)\right\}\right\rangle= & \int_{-\infty}^{\infty} d \omega \int_{-\infty}^{\infty} d \omega^{\prime} e^{i \omega \tau} e^{i \omega^{\prime} \tau^{\prime}} \\
& \times \chi_{\omega} \chi_{\omega^{\prime}}\left\langle\left\{\widetilde{\eta}(\omega), \widetilde{\eta}\left(\omega^{\prime}\right)\right\}\right\rangle \\
& =\frac{2 \hbar \gamma}{\pi} \int_{0}^{\infty} \frac{d k}{k}\left|\chi_{k}\right|^{2} \cos k\left(\tau-\tau^{\prime}\right) .
\end{aligned}
$$

\section{B. One accelerated detector: Unruh effect}

In the case of an accelerated detector moving on the trajectory $x(\tau)=a^{-1} \cosh a \tau, t(\tau)=a^{-1} \sinh a \tau$, and $s(\tau)=1$ ( $\tau$ being the proper time along the accelerated trajectory), the noise and dissipation kernels take the form

$$
\begin{aligned}
\widetilde{\nu}\left(\tau, \tau^{\prime}\right)= & \frac{e^{2}}{2 \pi} \int_{0}^{\infty} \frac{d k}{k} \cos \frac{k}{a}\left(\sinh a \tau-\sinh a \tau^{\prime}\right) \\
& \times \cos \frac{k}{a}\left(\cosh a \tau-\cosh a \tau^{\prime}\right), \\
\widetilde{\mu}\left(\tau, \tau^{\prime}\right)= & -\frac{e^{2}}{2 \pi} \int_{0}^{\infty} \frac{d k}{k} \sin \frac{k}{a}\left(\sinh a \tau-\sinh a \tau^{\prime}\right) \\
& \times \cos \frac{k}{a}\left(\cosh a \tau-\cosh a \tau^{\prime}\right) .
\end{aligned}
$$

These kernels can be decomposed into advanced and retarded parts, by writing, for example,

$$
\begin{aligned}
\widetilde{\nu}^{a}+\widetilde{\nu}^{r}= & \frac{e^{2}}{4 \pi} \int_{0}^{\infty} \frac{d k}{k}\left[\cos \frac{k}{a}\left(e^{a \tau}-e^{a \tau^{\prime}}\right)\right. \\
& \left.+\cos \frac{k}{a}\left(e^{-a \tau}-e^{-a \tau^{\prime}}\right)\right] .
\end{aligned}
$$

We can then use the changes of variables $k \rightarrow \frac{1}{2} k \exp \left[ \pm a / 2\left(\tau-\tau^{\prime}\right)\right]$ to obtain

$$
\widetilde{\nu}^{a}=\widetilde{\nu}^{r}=\frac{e^{2}}{4 \pi} \int_{0}^{\infty} \frac{d k}{k} \cos \left(\frac{k}{a} \sinh \frac{a}{2}\left(\tau-\tau^{\prime}\right)\right),
$$

showing that the noise felt by the accelerating detector is isotropic.

One can also make a similar simplification for the kernel $\tilde{\mu}$. These expressions can then be further simplified $[25,23]$ by means of the integral transform [27]

$$
e^{i k \sinh b}=\frac{2}{\pi} \int_{0}^{\infty} d \alpha \cos (b \alpha+i \pi \alpha / 2) K_{i \alpha}(k)
$$

and the formula [28]

$$
\int_{0}^{\infty} \frac{d k}{k} K_{i \alpha}(k)=\frac{\pi}{2 \alpha \sinh \left(\frac{\pi \alpha}{2}\right)},
$$

where $K_{i \alpha}(a)$ is a Bessel function of imaginary argument, to yield

$$
\begin{gathered}
\widetilde{\nu}\left(\tau, \tau^{\prime}\right)=\frac{e^{2}}{2 \pi} \int_{0}^{\infty} \frac{d k}{k} \operatorname{coth}\left(\frac{\pi k}{a}\right) \cos k\left(\tau-\tau^{\prime}\right), \\
\tilde{\mu}\left(\tau, \tau^{\prime}\right)=-\frac{e^{2}}{2 \pi} \int_{0}^{\infty} \frac{d k}{k} \sin k\left(\tau-\tau^{\prime}\right) .
\end{gathered}
$$

The noise experienced by the detector is thus stationary and the factor $\operatorname{coth}(\pi k / a)$ in the noise kernel shows that it is also thermal, at the Unruh temperature $k_{B} T=\hbar a / 2 \pi$ (we have chosen units such that $c=1$ ).

The dissipation kernel remains identical to that of the inertial detector. Based on the property that the two-point function of a free field on an accelerated trajectory evaluated in the Minkowski vacuum state is identical to the two-point function on an inertial trajectory evaluated in a thermal state at the Unruh temperature, this fact can be explained as follows. The dissipation kernel is proportional to the commutator of the free quantum field evaluated in whatever state the field is in. However, the commutator of a free field for any two points is just a $c$-number, hence its expectation value is independent of the state of the field. In particular, it does not distinguish between a zero temperature and a thermal state. So the dissipation kernel is identical to that in the inertial case. The anticommutator is, however, an operator whose expectation value depends on the state of the field, and therefore shows the familiar departure from the inertial case.

The Langevin equation for the detector coordinate is

$$
\frac{d^{2} Q}{d \tau^{2}}+\frac{e^{2}}{2} \frac{d Q}{d \tau}+\Omega_{0}^{2} Q=\frac{d \eta}{d \tau}
$$

with

$$
\left\langle\left\{\eta(\tau), \eta\left(\tau^{\prime}\right)\right\}\right\rangle=\hbar \widetilde{\nu}\left(\tau, \tau^{\prime}\right) .
$$

Similar to the inertial detector case, we find

$$
\widetilde{Q}(\omega)=\chi_{\omega} \widetilde{\eta}(\omega)
$$

Also,

$$
\begin{aligned}
\left\langle\left\{\widetilde{\eta}(\omega), \widetilde{\eta}\left(\omega^{\prime}\right)\right\}\right\rangle= & \frac{1}{4 \pi^{2}} \int_{-\infty}^{\infty} d \tau \int_{-\infty}^{\infty} d \tau^{\prime} e^{-i \omega \tau} \\
& \times e^{-i \omega^{\prime} \tau^{\prime}}\left\langle\left\{\eta(\tau), \eta\left(\tau^{\prime}\right)\right\}\right\rangle \\
= & \hbar \frac{\gamma}{\pi \omega} \operatorname{coth}\left(\frac{\pi \omega}{a}\right) \delta\left(\omega+\omega^{\prime}\right) .
\end{aligned}
$$


Combining the two equations,

$$
\begin{aligned}
\left\langle\left\{Q(\tau), Q\left(\tau^{\prime}\right)\right\}\right\rangle= & \int_{-\infty}^{\infty} d \omega \int_{-\infty}^{\infty} d \omega^{\prime} e^{i \omega \tau} e^{i \omega^{\prime} \tau^{\prime}} \\
& \times \chi_{\omega} \chi_{\omega^{\prime}}\left\langle\left\{\widetilde{\eta}(\omega), \widetilde{\eta}\left(\omega^{\prime}\right)\right\}\right\rangle \\
= & \frac{2 \hbar \gamma}{\pi} \int_{0}^{\infty} \frac{d k}{k}\left|\chi_{k}\right|^{2} \operatorname{coth}\left(\frac{\pi k}{a}\right) \cos k\left(\tau-\tau^{\prime}\right),
\end{aligned}
$$

with the impedance function $\chi_{k}$ as defined in the inertial case.

\section{Two inertial detectors: Self and mutual impedances}

We now consider the case of two detectors moving on the inertial trajectories $x_{1}\left(\tau_{1}\right)=-x_{0} / 2, \quad x_{2}\left(\tau_{2}\right)=x_{0} / 2$, and $t_{1}\left(\tau_{1}\right)=t_{2}\left(\tau_{2}\right)=\tau$, coupled to a scalar field initially in the Minkowski vacuum state, with coupling constants $e_{1,2}$. They are separated by a fixed coordinate distance $x_{0}$. As before, we will assume that both detectors have been forever switched on, i.e., $s_{i}(\tau)=1, i=1,2$.

It will be convenient to express the noise, dissipation, correlation, and propagation kernels as the real and imaginary parts of the functions $Z_{i j}$ defined earlier. Then, for the two-detector system, we obtain

$$
\begin{gathered}
Z_{11}\left(\tau, \tau^{\prime}\right)=\frac{e_{1}^{2}}{2 \pi} \int_{0}^{\infty} \frac{d k}{k} \exp \left[-i k\left(\tau-\tau^{\prime}\right)\right], \\
Z_{22}\left(\tau, \tau^{\prime}\right)=\frac{e_{2}^{2}}{2 \pi} \int_{0}^{\infty} \frac{d k}{k} \exp \left[-i k\left(\tau-\tau^{\prime}\right)\right], \\
Z_{12}\left(\tau, \tau^{\prime}\right)=Z_{21}\left(\tau, \tau^{\prime}\right) \\
=\frac{e_{1} e_{2}}{2 \pi} \int_{0}^{\infty} \frac{d k}{k} \exp \left[-i k\left(\tau-\tau^{\prime}\right)\right] \cos k x_{0} .
\end{gathered}
$$

The coupled Langevin equations for the system are

$$
\begin{aligned}
& \frac{d^{2} Q_{1}}{d \tau^{2}}+\frac{e_{1}^{2}}{2} \frac{d Q_{1}}{d \tau}+\left.\frac{e_{1} e_{2}}{2} \frac{d Q_{2}}{d \tau}\right|_{\tau-x_{0}}+\Omega_{1}^{2} Q_{1}=\frac{d \eta_{1}}{d \tau}, \\
& \frac{d^{2} Q_{2}}{d \tau^{2}}+\frac{e_{2}^{2}}{2} \frac{d Q_{2}}{d \tau}+\left.\frac{e_{1} e_{2}}{2} \frac{d Q_{1}}{d \tau}\right|_{\tau-x_{0}}+\Omega_{2}^{2} Q_{2}=\frac{d \eta_{2}}{d \tau},
\end{aligned}
$$

where $\tau-x_{0}$ is the retarded time between the two trajectories, and

$$
\left\langle\left\{\eta_{i}(\tau), \eta_{j}\left(\tau^{\prime}\right)\right\}\right\rangle=\hbar \widetilde{\nu}_{i j}\left(\tau-\tau^{\prime}\right) .
$$

As before, we define $\gamma_{1,2}=e_{1,2}^{2} / 4$, and introduce Fourier transforms to obtain the corresponding equations in frequency space. Then we obtain

$$
\widetilde{Q}_{1}(\omega)=\chi_{\omega}^{(1)} \widetilde{\eta}_{1}(\omega)-\frac{e_{1} e_{2}}{2} e^{-i \omega x_{0}} \chi_{\omega}^{(1)} \widetilde{Q}_{2}(\omega)
$$

$$
\widetilde{Q}_{2}(\omega)=\chi_{\omega}^{(2)} \widetilde{\eta}_{2}(\omega)-\frac{e_{1} e_{2}}{2} e^{-i \omega x_{0}} \chi_{\omega}^{(2)} \widetilde{Q}_{1}(\omega)
$$

where

$$
\chi_{\omega}^{(1),(2)}=i \omega\left(-\omega^{2}+\Omega_{1,2}^{2}+2 i \omega \gamma_{1,2}\right)^{-1} .
$$

The functions $\chi_{\omega}^{(1),(2)}$ are, of course, what the impedance of each detector would be in the absence of the other one. However, the effect of introducing a second detector is, as we shall see, to modify the "self-impedance" of each detector as well as introduce a "mutual impedance" which describes, for instance, the response of detector one to the force $\widetilde{\eta}_{2}$.

Indeed, plugging the equation for $\widetilde{Q}_{1}$ in the equation for $\widetilde{Q}_{2}$, we have

$$
\widetilde{Q}_{2}(\omega)=L_{22}(\omega) \widetilde{\eta}_{2}(\omega)+L_{21}(\omega) \widetilde{\eta}_{1}(\omega),
$$

where $L_{22}$ is the modified self-impedance of detector two because of the presence of detector one, and $L_{21}$ is the mutual impedance:

$$
\begin{aligned}
L_{22}(\omega)= & \chi_{\omega}^{(2)}\left(1-4 \gamma_{1} \gamma_{2} e^{-2 i \omega x_{0}} \chi_{\omega}^{(1)} \chi_{\omega}^{(2)}\right)^{-1}, \\
L_{21}(\omega)= & -2 \sqrt{\gamma_{1} \gamma_{2}} e^{-i \omega x_{0}} \chi_{\omega}^{(2)} \chi_{\omega}^{(1)} \\
& \times\left(1-4 \gamma_{1} \gamma_{2} e^{-2 i \omega x_{0}} \chi_{\omega}^{(1)} \chi_{\omega}^{(2)}\right)^{-1} .
\end{aligned}
$$

The impedances $L_{11}$ and $L_{12}$ and the corresponding equation for $\widetilde{Q}_{1}$ are obtained by an interchange of indices one and two in the above equations.

We note the symmetry

$$
L_{21}=L_{12} \text {. }
$$

The correlator $\left\langle\left\{Q_{i}(\omega), Q_{j}\left(\omega^{\prime}\right)\right\}\right\rangle, i, j=1,2$ is, therefore, obtained from Eq. (3.32) and its counterpart, as

$$
\begin{aligned}
\left\langle\left\{Q_{i}(\omega), Q_{j}\left(\omega^{\prime}\right)\right\}\right\rangle= & \sum_{\alpha=1}^{2} \sum_{\beta=1}^{2} L_{i \alpha}(\omega) L_{j \beta}\left(\omega^{\prime}\right) \\
& \times\left\langle\left\{\tilde{\eta}_{\alpha}(\omega), \tilde{\eta}_{\beta}\left(\omega^{\prime}\right)\right\}\right\rangle .
\end{aligned}
$$

The above equation is to be viewed as a generalization of (3.8) to the two-detector case.

Suppose we now wish to solve for the correlator of $Q_{2}$. Then, taking Fourier transforms as before and simplifying,

$$
\begin{aligned}
\left\langle\left\{Q_{2}(\tau), Q_{2}\left(\tau^{\prime}\right)\right\}\right\rangle= & \int_{-\infty}^{\infty} d \omega \int_{-\infty}^{\infty} d \omega^{\prime} e^{i \omega \tau} \\
& \times e^{i \omega^{\prime} \tau^{\prime}}\left\langle\left\{\widetilde{Q}_{2}(\omega), \widetilde{Q}_{2}\left(\omega^{\prime}\right)\right\}\right\rangle \\
= & \frac{2 \hbar \gamma_{2}}{\pi} \int_{0}^{\infty} \frac{d k}{k}\left|L_{22}(k)\right|^{2} \\
& \times\left[1+\gamma_{1}\left(4 \gamma_{1}\left|\chi_{k}^{(1)}\right|^{2}-\chi_{k}^{(1)}-\chi_{k}^{(1) *}\right)\right. \\
& -\gamma_{1}\left(\chi_{k}^{(1)} e^{\left.\left.-2 i k x_{0}+\chi_{k}^{(1) *} e^{2 i k x_{0}}\right)\right] .}\right.
\end{aligned}
$$


The second term in the square brackets vanishes as a consequence of the identity $\chi_{k}^{(1)}+\chi_{k}^{(1) *}=4 \gamma_{1}\left|\chi_{k}^{(1)}\right|^{2}$, which is a form of the fluctuation dissipation relation for detector one. The remaining terms simplify to yield

$$
\begin{aligned}
\left\langle\left\{Q_{2}(\tau), Q_{2}\left(\tau^{\prime}\right)\right\}\right\rangle= & \frac{2 \hbar \gamma_{2}}{\pi} \int_{0}^{\infty} \frac{d k}{k}\left|L_{22}(k)\right|^{2} \\
& \times\left[1-2 \gamma_{1}\left|\chi_{\omega}^{(1)}\right|^{2}\left(2 \gamma_{1} \cos 2 k x_{0}\right.\right. \\
& \left.\left.+\frac{\Omega_{1}^{2}-k^{2}}{k} \sin 2 k x_{0}\right)\right] \cos k\left(\tau-\tau^{\prime}\right) .
\end{aligned}
$$

As before, the correlator of $Q_{1}$ is obtained by interchanging the indices one and two in the above equation.

\section{UNIFORMLY ACCELERATED DETECTOR AND PROBE: ADVANCED AND RETARDED NOISES}

In this section, we first consider two detectors on arbitrary trajectories, with the following constraints: (a) both trajectories are everywhere timelike, (b) one of the trajectories possesses past and future event horizons, which are chosen to be the null lines $v=0$ and $u=0$, respectively, (c) the detector on the other trajectory is switched on at $u=0$ and this trajectory does not possess any future horizon.

Because of constraint (c), the second detector cannot causally influence the first one, and thus it functions as a probe in the field modified by the first detector.

Later in the analysis, we shall specify the trajectory of the detector with horizons as being a uniformly accelerated one. We shall continue to assume that the probe cannot causally influence the uniformly accelerated detector by means of the switching condition. If it were allowed to do so, this would lead to a deviation of the noise experienced by the uniformly accelerated detector from the precise thermal form.

We will label the detector with horizons as detector one and the probe as detector two. The switching condition $s_{2}\left(\tau_{2}\right)=\theta\left(u_{2}\left(\tau_{2}\right)\right)$ for the probe leads to a closed Langevin equation for detector one:

$$
\frac{d^{2} Q_{1}}{d \tau_{1}^{2}}+\frac{e_{1}^{2}}{2} \frac{d Q_{1}}{d \tau_{1}}+\Omega_{1}^{2} Q_{1}=\frac{d \eta_{1}}{d \tau_{1}}
$$

This is just a consequence of the fact that the trajectory of detector one lies outside the causal future of the probe. The arguments which lead to the above local form of dissipation or radiation reaction for a general timelike trajectory are outlined in the next section.

Introducing Fourier transforms and the impedance functions $\chi_{\omega}^{(1),(2)}$ as defined earlier, we have

$$
\widetilde{Q}_{1}(\omega)=\chi_{\omega}^{(1)} \widetilde{\eta}_{1}(\omega)
$$

Consider now the Langevin equation for detector two:

$$
\begin{aligned}
& \frac{d^{2} Q_{2}}{d \tau_{2}^{2}}-2 \int_{-\infty}^{\tau_{2}} d \tau_{2}^{\prime} \frac{d}{d \tau_{2}}\left[\theta\left(u_{2}\left(\tau_{2}\right)\right) \tilde{\mu}_{22}\left(\tau_{2}, \tau_{2}^{\prime}\right)\right] \frac{d Q_{2}}{d \tau_{2}^{\prime}} \\
& \quad-2 \int_{-\infty}^{t_{1}^{-1}\left(t_{2}\left(\tau_{2}\right)\right)} d \tau_{1}^{\prime} \frac{d}{d \tau_{2}}\left[\theta\left(u_{2}\left(\tau_{2}\right)\right) \tilde{\mu}_{21}\left(\tau_{2}, \tau_{1}^{\prime}\right)\right] \frac{d Q_{1}}{d \tau_{1}^{\prime}} \\
& \quad+\Omega_{2}^{2} Q_{2}=\frac{d}{d \tau_{2}}\left[\theta\left(v_{2}\left(\tau_{2}\right)\right) \eta_{2}\left(\tau_{2}\right)\right] .
\end{aligned}
$$

We find

$$
\begin{gathered}
\frac{d}{d \tau_{2}}\left(\tilde{\mu}_{22}\left(\tau_{2}, \tau_{2}^{\prime}\right)\right)=-2 \gamma_{2} \delta\left(\tau_{2}-\tau_{2}^{\prime}\right) \\
\frac{d}{d \tau_{2}}\left(\tilde{\mu}_{21}\left(\tau_{2}, \tau_{1}^{\prime}\right)\right)=-\sqrt{\gamma_{1} \gamma_{2}}\left[\frac{d v_{2}}{d \tau_{2}} \delta\left(v_{2}\left(\tau_{2}\right)-v_{1}\left(\tau_{1}^{\prime}\right)\right)\right. \\
\left.+\frac{d u_{2}}{d \tau_{2}} \delta\left(u_{2}\left(\tau_{2}\right)-u_{1}\left(\tau_{1}^{\prime}\right)\right)\right]
\end{gathered}
$$

where $\gamma_{1,2}$ are defined as in the two inertial detector cases. The second term in (4.5) vanishes identically because $u_{2}\left(\tau_{2}\right)>0$ and $u_{1}\left(\tau_{1}\right)<0 \quad(u=0$ is a future horizon for detector one). Since $v=0$ is a past horizon for detector one, we have $v_{1}\left(\tau_{1}^{\prime}\right)>0$ and the first term simplifies to yield

$$
\frac{d}{d \tau_{2}}\left(\tilde{\mu}_{21}\left(\tau_{1}, \tau_{2}^{\prime}\right)\right)=-\sqrt{\gamma_{1} \gamma_{2}} \frac{d \tau_{R}}{d \tau_{2}} \delta\left(\tau_{R}-\tau_{1}^{\prime}\right) \theta\left(v_{2}\left(\tau_{2}\right)\right)
$$

where we have defined the retarded time $\tau_{R}=v_{1}^{-1}\left(v_{2}\left(\tau_{2}\right)\right)$. This is well defined since it occurs only in expressions in which $v_{2}\left(\tau_{2}\right)>0$.

Thus, we obtain the dynamical equation for the probe, which depends, as expected, on $Q_{1}$ :

$$
\begin{array}{r}
\frac{d^{2} Q_{2}}{d \tau_{2}^{2}}+\frac{e_{2}^{2}}{2} \frac{d Q_{2}}{d \tau_{2}}+\Omega_{2}^{2} Q_{2}=\frac{d \eta_{2}}{d \tau_{2}}-\left.\frac{e_{1} e_{2}}{2} \frac{d \tau_{R}}{d \tau_{2}} \frac{d Q_{1}}{d \tau}\right|_{\tau_{R}}, \\
v_{2}\left(\tau_{2}\right)>0 .
\end{array}
$$

Consider the quantity

$$
F\left(\tau_{2}\right)=\frac{d \eta_{2}}{d \tau_{2}}-\left.\frac{e_{1} e_{2}}{2} \frac{d \tau_{R}}{d \tau_{2}} \frac{d Q_{1}}{d \tau}\right|_{\tau_{R}}
$$

which is a source term in the equation of motion for $Q_{2}$. The first part of $F$ is the usual stochastic force arising out of the fluctuations of the field in the vicinity of detector two, while the second part is the retarded force because of detector one. RSG correctly point out that these two forces are correlated. In the context of our formalism, these correlations are embodied in the correlation kernels $\widetilde{\nu}_{21}$ and $\widetilde{\nu}_{12}$.

Using the relation (4.2), we obtain

$$
\begin{aligned}
F\left(\tau_{2}\right)= & \frac{d \eta_{2}}{d \tau_{2}}-i \frac{e_{1} e_{2}}{4 \pi} \frac{d \tau_{R}}{d \tau_{2}} \int_{-\infty}^{\infty} d \omega \omega e^{i \omega \tau_{R}} \chi_{\omega}^{(1)} \int_{-\infty}^{\infty} \\
& \times d s \eta_{1}(s) e^{-i \omega s} .
\end{aligned}
$$


Consider the correlator of $F$ with the correlator of $\eta_{2}$ subtracted out. We have

$$
\begin{aligned}
\left\langle\left\{F\left(\tau_{2}\right), F\left(\tau_{2}^{\prime}\right)\right\}\right\rangle-\frac{d}{d \tau_{2}} \frac{d}{d \tau_{2}^{\prime}}\left\langle\left\{\eta_{2}\left(\tau_{2}\right), \eta_{2}\left(\tau_{2}^{\prime}\right)\right\}\right\rangle \\
=-\frac{i \hbar \sqrt{\gamma_{1} \gamma_{2}}}{\pi} \int_{-\infty}^{\infty} d \omega \omega \chi_{\omega}^{(1)} \int_{-\infty}^{\infty} d s e^{-i \omega s} \\
\quad \times\left[\frac{d \tau_{R}^{\prime}}{d \tau_{2}^{\prime}} e^{i \omega \tau_{R}^{\prime}} \frac{d}{d \tau_{2}} \widetilde{\nu}_{21}\left(\tau_{2}, s\right)+\frac{d \tau_{R}}{d \tau_{2}} e^{i \omega \tau_{R}} \frac{d}{d \tau_{2}^{\prime}} \widetilde{\nu}_{12}\left(s, \tau_{2}^{\prime}\right)\right] \\
-\frac{\hbar \gamma_{1} \gamma_{2}}{\pi^{2}} \frac{d \tau_{R}}{d \tau_{2}} \frac{d \tau_{R}^{\prime}}{d \tau_{2}^{\prime}} \int_{-\infty}^{\infty} d \omega \int_{-\infty}^{\infty} d \omega^{\prime} \omega \omega^{\prime} e^{i \omega \tau_{R}} \times e^{i \omega^{\prime} \tau_{R}^{\prime}} \\
\quad \times \chi_{\omega}^{(1)} \chi_{\omega^{\prime}}^{(1)} \int_{-\infty}^{\infty} d s \int_{-\infty}^{\infty} d s^{\prime} e^{-i \omega s} e^{-i \omega^{\prime} s^{\prime}} \widetilde{\nu}_{11}\left(s, s^{\prime}\right) .
\end{aligned}
$$

The kernels $\widetilde{\nu}_{21}$ and $\widetilde{\nu}_{12}$ separate into advanced and retarded parts. For the advanced parts,

$$
\begin{aligned}
\widetilde{\nu}_{21}^{a}\left(\tau_{2}, s\right) & =\frac{e_{1} e_{2}}{4 \pi} \int_{0}^{\infty} \frac{d k}{k} \cos k\left(v_{2}\left(\tau_{2}\right)-v_{1}(s)\right) \\
& =\frac{e_{1} e_{2}}{4 \pi} \int_{0}^{\infty} \frac{d k}{k} \cos k\left(v_{1}\left(\tau_{R}\right)-v_{1}(s)\right)=\frac{e_{2}}{e_{1}} \widetilde{\nu}_{11}^{a}\left(\tau_{R}, s\right)
\end{aligned}
$$

and similarly

$$
\widetilde{\nu}_{12}^{a}\left(s, \tau_{2}^{\prime}\right)=\frac{e_{2}}{e_{1}} \widetilde{\nu}_{11}^{a}\left(s, \tau_{R}^{\prime}\right) .
$$

The advanced parts of the correlations can therefore be constructed from the advanced part of the noise along the trajectory of detector one. With this simplification, we obtain

$$
\begin{aligned}
\left\langle\left\{F\left(\tau_{2}\right), F\left(\tau_{2}^{\prime}\right)\right\}\right\rangle-\frac{d}{d \tau_{2}} \frac{d}{d \tau_{2}^{\prime}}\left\langle\left\{\eta_{2}\left(\tau_{2}\right), \eta_{2}\left(\tau_{2}^{\prime}\right)\right\}\right\rangle \\
=-\frac{i \hbar \sqrt{\gamma_{1} \gamma_{2}} \frac{d \tau_{R}}{d} \frac{d \tau_{R}^{\prime}}{d \tau_{2}^{\prime}}}{\quad} \\
\quad \times\left[\int_{-\infty}^{\infty} d \omega \omega \chi_{\omega}^{(1)} \int_{-\infty}^{\infty} d s e^{-i \omega s}\right. \\
\quad \times\left\{e^{i \omega \tau_{R}^{\prime}} \frac{d}{d \tau_{R}} \widetilde{\nu}_{11}^{a}\left(\tau_{R}, s\right)+e^{i \omega \tau_{R}} \frac{d}{d \tau_{R}^{\prime}} \widetilde{\nu}_{11}^{a}\left(s, \tau_{R}^{\prime}\right)\right\} \\
-i \frac{\gamma_{1}}{\pi} \int_{-\infty}^{\infty} d \omega \int_{-\infty}^{\infty} d \omega^{\prime} \omega \omega^{\prime} e^{i \omega \tau_{R}} e^{i \omega^{\prime} \tau_{R}^{\prime}} \chi_{\omega}^{(1)} \chi_{\omega^{\prime}}^{(1)} \\
\left.\quad \times \int_{-\infty}^{\infty} d s \int_{-\infty}^{\infty} d s^{\prime} e^{-i \omega s} e^{-i \omega^{\prime} s^{\prime}} \widetilde{\nu}_{11}\left(s, s^{\prime}\right)\right]+(\text { r.p. }),
\end{aligned}
$$

where (r.p.) denotes the retarded part:

$$
\begin{aligned}
\text { (r.p.) }= & -\frac{i \hbar \sqrt{\gamma_{1} \gamma_{2}}}{\pi} \int_{-\infty}^{\infty} d \omega \omega \chi_{\omega}^{(1)} \int_{-\infty}^{\infty} d s e^{-i \omega s} \\
& \times\left[\frac{d \tau_{R}^{\prime}}{d \tau_{2}^{\prime}} e^{i \omega \tau_{R}^{\prime}} \frac{d}{d \tau_{2}} \widetilde{\nu}_{21}^{r}\left(\tau_{2}, s\right)\right. \\
& \left.+\frac{d \tau_{R}}{d \tau_{2}} e^{i \omega \tau_{R}} \frac{d}{d \tau_{2}^{\prime}} \widetilde{\nu}_{12}^{r}\left(s, \tau_{2}^{\prime}\right)\right]
\end{aligned}
$$

At this point, we specialize to the case when detector one is uniformly accelerated. Then, we have

$$
v_{1}\left(\tau_{1}\right)=a^{-1} e^{a \tau_{1}}, \quad u_{1}\left(\tau_{1}\right)=-a^{-1} e^{-a \tau_{1}} .
$$

As shown in Sec. III B, the noise $\widetilde{\nu}_{11}$ is thermal and isotropic. The retarded time $\tau_{R}=a^{-1} \ln \left[a v_{2}\left(\tau_{2}\right)\right]$. We may substitute for $\widetilde{\nu}_{11}$ in Eq. (4.13) and carry out the integrations over $s$ and $s^{\prime}$, to obtain

$$
\begin{aligned}
\left\langle\left\{F\left(\tau_{2}\right), F\left(\tau_{2}^{\prime}\right)\right\}\right\rangle-\frac{d}{d \tau_{2}} \frac{d}{d \tau_{2}^{\prime}}\left\langle\left\{\eta_{2}\left(\tau_{2}\right), \eta_{2}\left(\tau_{2}^{\prime}\right)\right\}\right\rangle \\
=-\frac{2 \hbar \gamma_{1} \gamma_{2}}{\pi} \frac{d \tau_{R}}{d \tau_{2}} \frac{d \tau_{R}^{\prime}}{d \tau_{2}^{\prime}} \int_{0}^{\infty} d k k \operatorname{coth} \frac{\pi k}{a} \cos k\left(\tau_{R}-\tau_{R}^{\prime}\right) \\
\quad \times\left(\chi_{k}^{(1)}+\chi_{k}^{(1) *}-4 \gamma_{1}\left|\chi_{k}^{(1)}\right|^{2}\right)+\text { (r.p.). }
\end{aligned}
$$

The first term in the above expression vanishes as a consequence of the identity $\chi_{k}^{(1)}+\chi_{k}^{(1) *}=4 \gamma_{1}\left|\chi_{k}^{(1)}\right|^{2}$, mentioned earlier. The only contribution to the excitation of the probe is, therefore, from the retarded parts of the correlations $\widetilde{\nu}_{12}$ and $\widetilde{\nu}_{21}$. This asymmetry between retarded and advanced parts is really a consequence of the choice of retarded boundary conditions in the formulation of the problem (the states of detector and field are assumed to be uncorrelated at past infinity) and the switching process at $u_{2}=0$. The vanishing of the first term in the above expression is a generalization of the cancellation obtained by RSG for a probe moving along an inertial trajectory. In order to study the retarded contribution in greater detail, it is desirable to simplify the correlations $\widetilde{\nu}_{12}^{r}$ and $\widetilde{\nu}_{21}^{r}$. The functions $Z_{12}^{r}$ and $Z_{21}^{r}$ take the form

$$
\begin{gathered}
Z_{12}^{r}\left(\tau_{1}, \tau_{2}^{\prime}\right)=\frac{e_{1} e_{2}}{4 \pi} \int_{0}^{\infty} \frac{d k}{k} \exp \left(\frac{i k}{a}\left[e^{-a \tau_{1}}+a u_{2}\left(\tau_{2}^{\prime}\right)\right]\right), \\
Z_{21}^{r}\left(\tau_{2}, \tau_{1}^{\prime}\right)=\frac{e_{1} e_{2}}{4 \pi} \int_{0}^{\infty} \frac{d k}{k} \exp \left(-\frac{i k}{a}\left[e^{-a \tau_{1}^{\prime}}+a u_{2}\left(\tau_{2}\right)\right]\right) .
\end{gathered}
$$

Introducing the Fourier transforms

$$
\begin{aligned}
\exp \left(\frac{i k}{a} e^{-a \tau_{1}}\right)= & \frac{1}{2 \pi a} \int_{-\infty}^{\infty} d \omega e^{-i \omega \tau_{1}} \Gamma\left(-\frac{i \omega}{a}\right) \\
& \times\left(\frac{k}{a}\right)^{i \omega / a} e^{\pi \omega /(2 a)}, \quad k>0,
\end{aligned}
$$




$$
\begin{aligned}
\exp \left(-\frac{i k}{a} e^{-a \tau_{1}}\right)= & \frac{1}{2 \pi a} \int_{-\infty}^{\infty} d \omega e^{i \omega \tau_{1}} \Gamma\left(\frac{i \omega}{a}\right) \\
& \times\left(\frac{k}{a}\right)^{-i \omega / a} e^{\pi \omega /(2 a)}, \quad k>0,
\end{aligned}
$$

and substituting in (4.19), we obtain

$$
\begin{aligned}
Z_{12}^{r}\left(\tau_{1}, \tau_{2}^{\prime}\right)= & \frac{e_{1} e_{2}}{8 \pi^{2} a} \int_{-\infty}^{\infty} d \omega e^{-i \omega \tau_{1}} e^{\pi \omega /(2 a)} \Gamma\left(-\frac{i \omega}{a}\right) \\
& \times \int_{0}^{\infty} \frac{d k}{k}\left(\frac{k}{a}\right)^{i \omega / a} \exp \left[i k u_{2}\left(\tau_{2}^{\prime}\right)\right] .
\end{aligned}
$$

The integral over $k$ can be evaluated in terms of gamma functions. Explicitly making use of the fact that $u_{2}\left(\tau_{2}^{\prime}\right)>0$, and simplifying,

$$
Z_{12}^{r}\left(\tau_{1}, \tau_{2}^{\prime}\right)=\frac{e_{1} e_{2}}{4 \pi} \int_{0}^{\infty} \frac{d \omega}{\omega} \frac{\cos \frac{\omega}{a}\left[a \tau_{1}+\ln a u_{2}\left(\tau_{2}^{\prime}\right)\right]}{\sinh \frac{\pi \omega}{a}}
$$

Similarly,

$$
Z_{21}^{r}\left(\tau_{2}, \tau_{1}^{\prime}\right)=\frac{e_{1} e_{2}}{4 \pi} \int_{0}^{\infty} \frac{d \omega}{\omega} \frac{\cos \frac{\omega}{a}\left[a \tau_{1}^{\prime}+\ln a u_{2}\left(\tau_{2}\right)\right]}{\sinh \frac{\pi \omega}{a}}
$$

Therefore, there is no contribution from the imaginary parts of $Z_{12}^{r}$ and $Z_{21}^{r}$ for $u_{2}\left(\tau_{2}^{\prime}\right)>0$, as obtained earlier [Eq. (4.5) leading to (4.6)].

Differentiating the above expressions with respect to $\tau_{2}^{\prime}$ and $\tau_{2}$, and substituting in the expression for (r.p.), one obtains, after carrying out the integration over $s$,

$$
\begin{aligned}
\left\langle\left\{F\left(\tau_{2}\right), F\left(\tau_{2}^{\prime}\right)\right\}\right\rangle-\frac{d}{d \tau_{2}} \frac{d}{d \tau_{2}^{\prime}}\left\langle\left\{\eta_{2}\left(\tau_{2}\right), \eta_{2}\left(\tau_{2}^{\prime}\right)\right\}\right\rangle \\
\equiv(\text { r.p. }) \\
=\frac{2 \hbar \gamma_{1} \gamma_{2}}{\pi} \int_{-\infty}^{\infty} \frac{d k k}{\sinh \frac{\pi k}{a}} \chi_{k}^{(1)} \\
\quad \times\left[\left(a^{2} u_{2}\left(\tau_{2}^{\prime}\right) v_{2}\left(\tau_{2}\right)\right)^{(i k / a)-1} \frac{d u_{2}}{d \tau_{2}^{\prime}} \frac{d v_{2}}{d \tau_{2}}\right. \\
\left.+\left(a^{2} u_{2}\left(\tau_{2}\right) v_{2}\left(\tau_{2}^{\prime}\right)\right)^{(i k / a)-1} \frac{d u_{2}}{d \tau_{2}} \frac{d v_{2}}{d \tau_{2}^{\prime}}\right]
\end{aligned}
$$

The coincidence limit of the above expression yields the fluctuations of the random force acting on the probe: defining $\delta F(\tau)=F(\tau)-(d / d \tau) \eta_{2}(\tau)$, we obtain

$$
\begin{aligned}
\left\langle\delta F^{2}\left(\tau_{2}\right)\right\rangle= & \frac{2 \hbar \gamma_{1} \gamma_{2}}{\pi} \frac{d u_{2}}{d \tau_{2}} \frac{d v_{2}}{d \tau_{2}} \int_{-\infty}^{\infty} \frac{d k k}{\sinh \frac{\pi k}{a}} \\
& \times \chi_{k}^{(1)}\left(a^{2} u_{2}\left(\tau_{2}\right) v_{2}\left(\tau_{2}\right)\right)^{(i k / a)-1} .
\end{aligned}
$$

The fluctuations are thus suppressed in the limit of large $u_{2} v_{2}=t_{2}^{2}-x_{2}^{2}$. For a probe trajectory without any horizon, this is the limit in which the probe trajectory approaches future timelike infinity, which verifies that the effect of the accelerated oscillator on the field is ascribed to polarization rather than radiation (see also [13]). A radiation field is expected to persist at future infinity.

Let us now turn to the question of the response of the probe. To obtain this, we will need to specify a particular form of trajectory for the probe as well. We will consider the simple inertial trajectory $x_{2}\left(\tau_{2}\right)=0, t_{2}\left(\tau_{2}\right)=\tau_{2}$, switched on at $\tau_{2}=0$. Then Eq. (4.23) gives

$$
\begin{aligned}
& \left\langle\left\{F\left(\tau_{2}\right), F\left(\tau_{2}^{\prime}\right)\right\}\right\rangle-\frac{d}{d \tau_{2}} \frac{d}{d \tau_{2}^{\prime}}\left\langle\left\{\eta_{2}\left(\tau_{2}\right), \eta_{2}\left(\tau_{2}^{\prime}\right)\right\}\right\rangle \\
& =\frac{2 \hbar \gamma_{1} \gamma_{2}}{\pi} \int_{-\infty}^{\infty} \frac{d k k}{\sinh \frac{\pi k}{a}} \chi_{k}^{(1)}\left(a \tau_{2}\right)^{(i k / a)-1}\left(a \tau_{2}^{\prime}\right)^{(i k / a)-1} .
\end{aligned}
$$

Owing to the switching process at $\tau_{2}=0$, the relation between $\widetilde{Q}_{2}$ and $\widetilde{F}$ (the Fourier transforms of $Q_{2}$ and $F$ ) is a nonlocal one in frequency space, because of transient effects. However, if we restrict our attention to the late time behavior of detector two, we obtain from Eq. (4.7) a local relation of the form

$$
\widetilde{Q}_{2}(\omega)=\frac{\chi_{\omega}^{(2)}}{i \omega} \widetilde{F}(\omega)
$$

where

$$
\widetilde{F}(\omega)=\frac{1}{2 \pi} \int_{0}^{\infty} d \tau e^{-i \omega \tau} F(\tau)
$$

In the above expression, the lower limit of the $\tau$ integration is zero, corresponding to the step function $\theta(\tau)$ multiplying $F$ which enforces the switching condition.

The correlator of $\widetilde{Q}_{2}$ is, therefore, given by

$$
\left\langle\left\{\widetilde{Q}_{2}(\omega), \widetilde{Q}_{2}\left(\omega^{\prime}\right)\right\}\right\rangle=-\frac{\chi_{\omega}^{(2)} \chi_{\omega^{\prime}}^{(2)}}{\omega \omega^{\prime}}\left\langle\left\{\widetilde{F}(\omega), \widetilde{F}\left(\omega^{\prime}\right)\right\}\right\rangle
$$

We have already obtained the difference of the correlator of $F$ from its value in the absence of the accelerating detector, one. Thus, we have

$$
\begin{aligned}
\left\langle\left\{\widetilde{Q}_{2}(\omega), \widetilde{Q}_{2}\left(\omega^{\prime}\right)\right\}\right\rangle-\left\langle\left\{\widetilde{Q}_{2}^{(0)}(\omega), \widetilde{Q}_{2}^{(0)}\left(\omega^{\prime}\right)\right\}\right\rangle \\
=-\frac{\chi_{\omega}^{(2)} \chi_{\omega^{\prime}}^{(2)}}{4 \pi^{2} \omega \omega^{\prime}} \int_{0}^{\infty} d \tau \int_{0}^{\infty} d \tau^{\prime} e^{-i \omega \tau} e^{-i \omega^{\prime} \tau^{\prime}} \\
\quad \times\left[\left\langle\left\{F(\tau), F\left(\tau^{\prime}\right)\right\}\right\rangle-\frac{d}{d \tau} \frac{d}{d \tau^{\prime}}\left\langle\left\{\eta_{2}(\tau), \eta_{2}\left(\tau^{\prime}\right)\right\}\right\rangle\right]
\end{aligned}
$$

where the superscript (0) on $Q_{2}$ refers to its value in the absence of the accelerating detector. Performing the integrations over $\tau$ and $\tau^{\prime}$, we obtain 


$$
\begin{aligned}
&\left\langle\left\{\widetilde{Q}_{2}(\omega), \widetilde{Q}_{2}\left(\omega^{\prime}\right)\right\}\right\rangle-\left\langle\left\{\widetilde{Q}_{2}^{(0)}(\omega), \widetilde{Q}_{2}^{(0)}\left(\omega^{\prime}\right)\right\}\right\rangle \\
&=- \frac{\hbar \gamma_{1} \gamma_{2}}{2 \pi^{3} a^{4}} \frac{\chi_{\omega}^{(2)} \chi_{\omega^{\prime}}^{(2)}}{\omega \omega^{\prime}} \int_{-\infty}^{\infty} \frac{d k k}{\sinh \frac{\pi k}{a}} \chi_{k}^{(1)} \Gamma^{2}\left(\frac{i k}{a}\right) \\
& \times\left|\frac{\omega \omega^{\prime}}{a^{2}}\right|^{-(i k / a)-1}\left[e^{\pi k / 2 a} \theta(\omega)+e^{-\pi k / 2 a} \theta(-\omega)\right] \\
& \times\left[e^{\pi k / 2 a} \theta\left(\omega^{\prime}\right)+e^{-\pi k / 2 a} \theta\left(-\omega^{\prime}\right)\right] .
\end{aligned}
$$

The step functions which distinguish positive and negative frequencies in the above expression are an artefact of the switching process.

\section{FLUCTUATION-DISSIPATION AND CORRELATION-PROPAGATION RELATIONS}

In this section we construct the fluctuation-dissipation relations for the detector system and extend this construction to obtain a new set of relations, which we call the correlationpropagation relations for trajectories without any event horizon. These relations are a simple consequence of the analytic properties of the massless free field two-point function. We also discuss these relations in the context of the model of a uniformly accelerated detector and probe.

Consider first the fluctuation-dissipation relation for a quantum Brownian particle in a heat bath [22]. This can be expressed as a linear, nonlocal relation between the noise $\widetilde{\nu}(s)$ and dissipation $\widetilde{\mu}(s)$ kernels. Defining $\widetilde{\gamma}$ by

$$
\tilde{\mu}(s)=\frac{d \tilde{\gamma}}{d s}(s),
$$

the finite temperature fluctuation-dissipation relation is

$$
\widetilde{\nu}(s)=\int_{-\infty}^{\infty} d s^{\prime} K\left(s-s^{\prime}\right) \widetilde{\gamma}\left(s^{\prime}\right),
$$

where

$$
K\left(s-s^{\prime}\right)=\int_{0}^{\infty} \frac{k d k}{\pi} \operatorname{coth}\left(\frac{\beta k \hbar}{2}\right) \cos k\left(s-s^{\prime}\right)
$$

is a universal kernel, independent of the spectral density of the bath. In particular, the kernel $K$ is independent of the coupling constant $e$. Such a fluctuation-dissipation relation holds for the uniformly accelerated detector (with temperature given by the Unruh temperature) and the inertial detector (with zero temperature). It was derived in [22] in the context of a quantum Brownian model with bilinear coupling between bath (field) and particle (detector). In such a model $\widetilde{\gamma}$ is indeed the quantity which characterizes dissipation in the effective Langevin equation for the particle.

In the context of the minimally coupled model, however, we find it suitable to define $\tilde{\gamma}$ as

$$
\widetilde{\gamma}(s)=-\frac{d}{d s} \tilde{\mu}(s)
$$

as this is the quantity which directly appears in the dissipative term of the Langevin equation derived above. The fluctuation-dissipation relation then takes the form (5.3) with $K$ defined as

$$
K\left(s-s^{\prime}\right)=\int_{0}^{\infty} \frac{d k}{\pi k} \operatorname{coth}\left(\frac{\beta k \hbar}{2}\right) \cos k\left(s-s^{\prime}\right) .
$$

An important aspect of either form of the fluctuationdissipation relation is that the noise and dissipation kernels, and consequently $K$, are stationary; i.e., they are functions of $s-s^{\prime}$ alone.

We wish to investigate whether a suitable generalization of the above relation holds for the full $N$-detector system. To this end, we assume that the detector trajectories are everywhere timelike and consider first only the kernels $Z_{i i}$ as they characterize noise and dissipation in the dynamics of the detectors $i$. We also assume that the detectors are switched on forever, thus excluding transient effects because of the switching process. Using advanced and retarded null coordinates introduced earlier, we define

$$
\begin{aligned}
\widetilde{\gamma}_{i i}\left(\tau_{i}, \tau_{i}^{\prime}\right)= & -\frac{d}{d \tau_{i}} \tilde{\mu}_{i i}\left(\tau_{i}, \tau_{i}^{\prime}\right) \\
= & \frac{e_{i}^{2}}{4}\left[\delta\left(v_{i}\left(\tau_{i}\right)-v_{i}\left(\tau_{i}^{\prime}\right)\right) \frac{d v_{i}}{d \tau_{i}}\right. \\
& \left.+\delta\left(u_{i}\left(\tau_{i}\right)-u_{i}\left(\tau_{i}^{\prime}\right)\right) \frac{d u_{i}}{d \tau_{i}}\right] \\
= & \widetilde{\gamma}_{i i}^{a}\left(\tau_{i}, \tau_{i}^{\prime}\right)+\widetilde{\gamma}_{i i}^{r}\left(\tau_{i}, \tau_{i}^{\prime}\right),
\end{aligned}
$$

denoting the advanced and retarded parts of the kernel $\tilde{\gamma}$.

The timelike property of the trajectories implies that $\left|d x_{i} / d t_{i}\right|<1$. Together with the fact that $t_{i}\left(\tau_{i}\right)$ are increasing functions of $\tau_{i}$, this implies that $d u_{i} / d \tau_{i}$ and $d v_{i} / d \tau_{i}$ are necessarily positive. It also implies that the functions $u_{i}\left(\tau_{i}\right)$ and $v_{i}\left(\tau_{i}\right)$ have unique inverses, if they exist. This can be proved by way of contradiction: assume that $u_{i}\left(\tau_{i}\right)=u_{i}\left(\tau_{i}^{\prime}\right)$ for some $\tau_{i} \neq \tau_{i}^{\prime}$. Then we have $x_{i}\left(\tau_{i}\right)-x_{i}\left(\tau_{i}^{\prime}\right)$ $=t_{i}\left(\tau_{i}\right)-t_{i}\left(\tau_{i}^{\prime}\right)$, which means that the points $\tau_{i}$ and $\tau_{i}^{\prime}$ have lightlike separation. This contradicts the fact that the trajectory is everywhere timelike. The uniqueness of $v_{i}^{-1}$ is shown in the same way.

These two properties lead to the simplification in the expression for $\widetilde{\gamma}_{i i}$

$$
\widetilde{\gamma}_{i i}\left(\tau_{i}, \tau_{i}^{\prime}\right)=\frac{e_{i}^{2}}{2} \delta\left(\tau_{i}-\tau_{i}^{\prime}\right)
$$

Thus, we see that, for an arbitrary trajectory, the dissipation or radiation reaction kernel has the same form and is always local. This fact has been used in obtaining the dissipative term in the equations of motion for the accelerated detector and probe (4.1) and (4.7).

The fluctuation-dissipation relation now follows in a straightforward manner:

$$
\widetilde{\nu}_{i i}\left(\tau_{i}, \tau_{i}^{\prime}\right)=\int_{-\infty}^{\infty} d s K_{i}\left(\tau_{i}, s\right) \widetilde{\gamma}_{i i}\left(s, \tau_{i}^{\prime}\right),
$$


where

$$
\begin{aligned}
K_{i}\left(\tau_{i}, s\right)= & K_{i}^{a}\left(\tau_{i}, s\right)+K_{i}^{r}\left(\tau_{i}, s\right) \\
= & \int_{0}^{\infty} \frac{d k}{2 \pi k}\left[\cos k\left(v_{i}\left(\tau_{i}\right)-v_{i}(s)\right)\right. \\
& \left.+\cos k\left(u_{i}\left(\tau_{i}\right)-u_{i}(s)\right)\right] .
\end{aligned}
$$

We now ask whether a similar relation holds between the real and imaginary parts of $Z_{i j}, i \neq j$. This would not be a fluctuation-dissipation relation in the usual sense, as the real part of $Z_{i j}$ describes correlations of the field between points on different trajectories rather than fluctuations, and its imaginary part describes the propagation of radiation between one detector and the other, rather than dissipation. We will call such relations "correlation-propagation'" relations.

If points on different trajectories have spacelike separations, the relevant $\widetilde{\gamma}_{i j}$ (defined as $-d \tilde{\mu}_{i j} / d \tau_{i}$ ) will vanish as a consequence of the vanishing of the commutator of a free field for points at spacelike separations. This is simply an expression of causality in the detector dynamics. However, the corresponding correlation $\widetilde{\nu}_{i j}$ need not vanish, and hence there cannot be a general relation between these two kernels. Such a situation is realized most clearly, for example, in the case of two uniformly accelerating detectors, one in the right and the other in the left Rindler wedge. The trajectories, although individually timelike, are spacelike separated everywhere. The corresponding $\widetilde{\gamma}_{12}$ and $\widetilde{\gamma}_{21}$ will therefore vanish identically. However, $\widetilde{\nu}_{12}$ and $\widetilde{\nu}_{21}$ will remain nonzero, reflecting the highly correlated nature of the Minkowski vacuum state.

If, however, none of the detector trajectories possesses past or future horizon (in Minkowski space this is true, in particular, for geodesic trajectories, but not only for geodesic trajectories), then each of them will lie completely within the causal future of the others. In that case, we can obtain correlation-propagation relations relating separately the advanced and retarded correlations to their "propagating", counterparts. These relations follow from the fluctuationdissipation relations along single trajectories derived above, essentially by a method of geometric construction: defining $\widetilde{\gamma}_{i j}^{a}=-d \tilde{\mu}_{i j}^{a} / d \tau_{i}$ and similarly $\tilde{\gamma}_{i j}^{r}$, we have

$$
\widetilde{\gamma}_{i j}^{a}\left(\tau_{i}, \tau_{j}^{\prime}\right)=\frac{e_{i} e_{j}}{4} \delta\left(v_{i}\left(\tau_{i}\right)-v_{j}\left(\tau_{j}^{\prime}\right)\right) \frac{d v_{i}}{d \tau_{i}} .
$$

Since the trajectory $i$ does not possess any horizon, the null coordinates $u_{i}$ and $v_{i}$ range from $-\infty$ to $\infty$. Thus, the functions $v_{i} v_{i}^{-1}$ and $u_{i} u_{i}^{-1}$ are identity functions over the entire real line. Then we obtain, similar to Eq. (5.6),

$$
\begin{aligned}
\widetilde{\gamma}_{i j}^{a}\left(\tau_{i}, \tau_{j}^{\prime}\right) & =\frac{e_{i} e_{j}}{4} \delta\left[\tau_{i}-v_{i}^{-1}\left(v_{j}\left(\tau_{j}^{\prime}\right)\right)\right] \\
& =\frac{e_{j}}{e_{i}} \widetilde{\gamma}_{i i}^{a}\left[\tau_{i}, v_{i}^{-1}\left(v_{j}\left(\tau_{j}^{\prime}\right)\right)\right]
\end{aligned}
$$

and

$$
\widetilde{\gamma}_{i j}^{r}\left(\tau_{i}, \tau_{j}^{\prime}\right)=\frac{e_{j}}{e_{i}} \widetilde{\gamma}_{i i}^{r}\left[\tau_{i}, u_{i}^{-1}\left(u_{j}\left(\tau_{j}^{\prime}\right)\right)\right]
$$

The correlations $\widetilde{\nu}_{i j}$ may be constructed from the noises $\widetilde{\nu}_{i i}$ in an identical manner:

$$
\begin{aligned}
\widetilde{\nu}_{i j}^{a}\left(\tau_{i}, \tau_{j}^{\prime}\right) & =\frac{e_{i} e_{j}}{4 \pi} \int_{0}^{\infty} \frac{d k}{k} \cos k\left[v_{i}\left(\tau_{i}\right)-v_{i}\left(v_{i}^{-1} v_{j}\left(\tau_{j}^{\prime}\right)\right)\right] \\
& =\frac{e_{j}}{e_{i}} \widetilde{\nu}_{i i}^{a}\left(\tau_{i}, v_{i}^{-1} v_{j}\left(\tau_{j}^{\prime}\right)\right)
\end{aligned}
$$

where we have inserted the identity function $v_{i} v_{i}^{-1}$ in the first step. Also,

$$
\widetilde{\nu}_{i j}^{r}\left(\tau_{i}, \tau_{j}^{\prime}\right)=\frac{e_{j}}{e_{i}} \widetilde{\nu}_{i i}^{r}\left(\tau_{i}, u_{i}^{-1} u_{j}\left(\tau_{j}^{\prime}\right)\right)
$$

These two sets of constructions for the propagation and correlation kernels in terms of the dissipation and noise kernels enable us to write down the correlation-propagation relations simply by invoking the fluctuation-dissipation relations (5.8) as they separately apply to the advanced and retarded parts of the noise and dissipation along single trajectories:

$$
\widetilde{\nu}_{i j}^{a, r}\left(\tau_{i}, \tau_{j}^{\prime}\right)=\int_{-\infty}^{\infty} d s K_{i}^{a, r}\left(\tau_{i}, s\right) \widetilde{\gamma}_{i j}^{a, r}\left(s, \tau_{j}^{\prime}\right),
$$

$K_{i}^{a}$ and $K_{i}^{r}$ being defined earlier (5.9). Since the quantities $\tilde{\gamma}_{i j}$ are really just $\delta$ functions and the quantities $K_{i}^{a, r}$ are proportional to $\widetilde{\nu}_{i i}^{a, r}$, these relations can be equivalently viewed as constructions of the correlations $\widetilde{\nu}_{i j}$ from the noises $\widetilde{\nu_{i i}}$.

The above relations hold for trajectories without any event horizon. In the example of the uniformly accelerated detector and probe, the uniformly accelerated detector trajectory does possess event horizons. This manifests in the property that the range of $u_{1}$ is restricted to $(-\infty, 0)$ and the range of $v_{1}$ to $(0, \infty)$. The probe trajectory, on the other hand, will be chosen to be free of any horizon. We will also now assume that the probe is switched on forever. Then, we can construct the correlations $\widetilde{\nu}_{21}$ and the quantities $\widetilde{\gamma}_{21}$ from $\widetilde{\nu}_{22}$ and $\widetilde{\gamma}_{22}$ exactly as described above, and obtain the corresponding correlation-propagation relations:

$$
\widetilde{\nu}_{21}^{a, r}\left(\tau_{2}, \tau_{1}^{\prime}\right)=\int_{-\infty}^{\infty} d s K_{2}^{a, r}\left(\tau_{2}, s\right) \widetilde{\gamma}_{21}^{a, r}\left(s, \tau_{1}^{\prime}\right)
$$

This simply follows by invoking the fluctuation-dissipation relation along the probe trajectory, as described above. However, it is of greater interest to know whether such relations would follow from the fluctuation-dissipation relation along the uniformly accelerated trajectory. As explained, this will not be completely possible because the accelerated trajectory possesses horizons. This difficulty shows up when one tries to write down a relation of the form (5.16) for the quantities $\widetilde{\nu}_{12}$ and $\widetilde{\gamma}_{12}$. To do this, we first express the functions $Z_{i j}$ in a different form. This was done in Sec. IV [see the steps leading from (3.54)-(3.58)] for the restricted case $u_{2}\left(\tau_{2}\right)>0, v_{2}\left(\tau_{2}\right)>0$. If we remove this restriction, we find 


$$
\begin{aligned}
Z_{12}^{a}\left(\tau_{1}, \tau_{2}^{\prime}\right)= & \frac{e_{1} e_{2}}{4 \pi} \int_{0}^{\infty} \frac{d \omega}{\omega}\left[\operatorname{coth}\left(\frac{\pi \omega}{a}\right) \cos \frac{\omega}{a}\left(a \tau_{1}-\ln \left|a v_{2}\left(\tau_{2}^{\prime}\right)\right|\right) \theta\left(v_{2}\left(\tau_{2}^{\prime}\right)\right)+\frac{\cos \frac{\omega}{a}\left(a \tau_{1}-\ln \left|a v_{2}\left(\tau_{2}^{\prime}\right)\right|\right)}{\sinh \frac{\pi \omega}{a}} \theta\left(-v_{2}\left(\tau_{2}^{\prime}\right)\right)\right. \\
& \left.-i \sin \frac{\omega}{a}\left(a \tau_{1}-\ln \left|a v_{2}\left(\tau_{2}^{\prime}\right)\right|\right) \theta\left(v_{2}\left(\tau_{2}^{\prime}\right)\right)\right] \\
Z_{12}^{r}\left(\tau_{1}, \tau_{2}^{\prime}\right)= & \frac{e_{1} e_{2}}{4 \pi} \int_{0}^{\infty} \frac{d \omega}{\omega}\left[\operatorname{coth}\left(\frac{\pi \omega}{a}\right) \cos \frac{\omega}{a}\left(a \tau_{1}+\ln \left|a u_{2}\left(\tau_{2}^{\prime}\right)\right|\right) \theta\left(-u_{2}\left(\tau_{2}^{\prime}\right)\right)+\frac{\cos \frac{\omega}{a}\left(a \tau_{1}+\ln \left|a u_{2}\left(\tau_{2}^{\prime}\right)\right|\right)}{\sinh \frac{\pi \omega}{a}} \theta\left(u_{2}\left(\tau_{2}^{\prime}\right)\right)\right. \\
& \left.-i \sin \frac{\omega}{a}\left(a \tau_{1}+\ln \left|a u_{2}\left(\tau_{2}^{\prime}\right)\right|\right) \theta\left(-u_{2}\left(\tau_{2}^{\prime}\right)\right)\right]
\end{aligned}
$$

$Z_{21}$ can be expressed in a similar way. From the above, we see that the advanced (retarded) correlation for $v_{2}>0$ $\left(u_{2}<0\right)$ has a thermal form, because these correlations can be constructed simply from the noise along the accelerated trajectory. We are, therefore, able to write down a correlation-propagation relation for this part of the correlations alone. This takes the form

$$
\begin{gathered}
\theta\left(v_{2}\left(\tau_{2}^{\prime}\right)\right) \widetilde{\nu}_{12}^{a}\left(\tau_{1}, \tau_{2}^{\prime}\right)+\theta\left(-u_{2}\left(\tau_{2}^{\prime}\right)\right) \tilde{\nu}_{12}^{r}\left(\tau_{1}, \tau_{2}^{\prime}\right) \\
=\int_{-\infty}^{\infty} d s K_{1}\left(\tau_{1}, s\right) \widetilde{\gamma}_{12}\left(s, \tau_{2}^{\prime}\right),
\end{gathered}
$$

where

$$
\begin{aligned}
\tilde{\gamma}_{12}\left(\tau_{1}, \tau_{2}^{\prime}\right)= & -\frac{d \tilde{\mu}_{12}}{d \tau_{1}} \\
= & \frac{e_{1} e_{2}}{4}\left[\delta\left(\tau_{1}-a^{-1} \ln \left|a v_{2}\left(\tau_{2}^{\prime}\right)\right|\right) \theta\left(v_{2}\left(\tau_{2}^{\prime}\right)\right)\right. \\
& \left.+\delta\left(\tau_{1}+a^{-1} \ln \left|a u_{2}\left(\tau_{2}^{\prime}\right)\right|\right) \theta\left(-u_{2}\left(\tau_{2}^{\prime}\right)\right)\right]
\end{aligned}
$$

and

$$
K_{1}\left(\tau_{1}, s\right)=\int_{0}^{\infty} \frac{d k}{\pi k} \operatorname{coth} \frac{\pi k}{a} \cos k\left(\tau_{1}-s\right)
$$

The single relation (5.19), as opposed to separate relations between the advanced and retarded parts, is a consequence of the fact that the thermal noise is isotropic and therefore contains equal contributions from advanced and retarded parts.

In the context of the analysis of Sec. IV, where the probe is switched on at $u_{2}=0,(5.19)$ becomes

$$
\widetilde{\nu}_{12}^{a}\left(\tau_{1}, \tau_{2}^{\prime}\right)=\int_{-\infty}^{\infty} d s K_{1}^{a}\left(\tau_{1}, s\right) \widetilde{\gamma}_{12}^{a}\left(s, \tau_{2}^{\prime}\right)
$$

Viewed as a construction of $\widetilde{\nu}_{12}^{a}$ from $\widetilde{\nu}_{11}^{a}$, this relation lies at the heart of the RSG cancellation in (4.16). Viewed alternatively as an extension of the thermal fluctuation-dissipation relation on the uniformly accelerated trajectory, it thus places the role of thermal equilibrium in the RSG cancellation on firmer ground.

We now turn to the part of the correlations which do not partake in the correlation-propagation relation above. These are the advanced (retarded) correlations for $v_{2}<0\left(u_{2}>0\right)$, containing the arcsinh factors, and are not expressible in terms of the noise along the accelerated trajectory. Rather, they represent true correlations across the future (past) horizon. If we specialize to the case $u_{2}>0$ as in Sec. IV, then these are exactly the correlations which contribute to the excitation of the probe in the guise of (r.p.), Eq. (4.23). The probe may, therefore, be said to be excited by free field correlations across the future horizon.

If we specialize to the simple probe trajectory $x_{2}\left(\tau_{2}\right)=0, t_{2}\left(\tau_{2}\right)=\tau_{2}$, then we have $u_{2}\left(\tau_{2}\right)=v_{2}\left(\tau_{2}\right)=\tau_{2}$ and the expressions (5.18) for $Z_{12}$ acquire a symmetric form. In this special case, we can write down a correlationpropagation relation for the entire kernel $\widetilde{\nu}_{12}$, by relating the advanced part of the correlations across the horizon to the retarded part of the propagation kernel, and vice versa: we then have

$$
\begin{aligned}
\widetilde{\gamma}_{12}\left(\tau_{1}, \tau_{2}^{\prime}\right)= & \frac{e_{1} e_{2}}{4}\left[\delta\left(\tau_{1}-a^{-1} \ln \left|a \tau_{2}^{\prime}\right|\right) \theta\left(\tau_{2}^{\prime}\right)\right. \\
& \left.+\delta\left(\tau_{1}+a^{-1} \ln \left|a \tau_{2}^{\prime}\right|\right) \theta\left(-\tau_{2}^{\prime}\right)\right]
\end{aligned}
$$

Choosing

$K_{1}^{\prime}\left(\tau_{1}, \tau_{1}^{\prime}\right)=\int_{0}^{\infty} \frac{d k}{\pi k}\left[\operatorname{coth} \frac{\pi k}{a} \cos k\left(\tau_{1}-\tau_{1}^{\prime}\right)+\frac{\cos k\left(\tau_{1}+\tau_{1}^{\prime}\right)}{\sinh \frac{\pi k}{a}}\right]$,

we obtain

$$
\widetilde{\nu}_{12}\left(\tau_{1}, \tau_{2}^{\prime}\right)=\int_{-\infty}^{\infty} d s K_{1}^{\prime}\left(\tau_{1}, s\right) \widetilde{\gamma}_{12}\left(s, \tau_{2}^{\prime}\right)
$$

as a correlation-propagation relation in this special case. The above relation cannot be geometrically constructed from the fluctuation-dissipation relation along the single accelerated trajectory. So far, we have not been able to show the existence of such a relation in more general cases. The extra piece in the interpolating kernel $K_{1}^{\prime}$ comes from correlations across the horizon, as explained earlier. 


\section{SUMMARY AND DISCUSSION}

To summarize our work and findings, we have presented a general formalism to treat an arbitrary number of detectors modelled as oscillators in arbitrary kinematic states, and minimally coupled to a massless scalar field in $1+1$ dimensions. In this approach, the scalar field has been integrated out and the detector dynamics is described by a reduced set of effective semiclassical stochastic equations. These equations, nonetheless, contain the full quantum dynamics of the field. Our treatment can be extended to massive fields and higher dimensions by making appropriate changes in the two-point functions $Z_{i j}$.

We studied four examples, starting with a single inertial and uniformly accelerated detector, mainly to illustrate the new description, and culminating in the treatment of a uniformly accelerated oscillator and a second oscillator which functions as a probe. We show that there exist fluctuationdissipation relations relating the fluctuations of the stochastic forces on the detectors to the dissipative forces. We discover a related set of correlation-propagation relations between the correlations of stochastic forces on different detectors and the retarded and advanced parts of the radiation mediated by them.

In the analysis of two inertial detectors, we find that the change in the state of the field because of the coupling with either detector modifies the impedance functions of both detectors, and hence their dissipative properties. Also, this coupling introduces a mutual impedance which describes the change in the response of one detector because of the fluctuations of the field in the vicinity of the other one. The field fluctuations (noise) in this case are relatively trivial, and nontrivial effects can be ascribed mainly to the impedance functions.

In the case of the accelerated detector and the probe, on the other hand, the noise because of field fluctuations and the field correlations between the two trajectories play a dominant role. Since the probe cannot causally influence the accelerated detector, the dissipative features of this problem are relatively trivial. Here, we find that most of the terms contributing to the response of the probe cancel out, leaving behind a contribution that arises purely from field correlations across the horizon. This cancellation was earlier pointed out $[11,13]$ to be a consequence of the identity $\chi_{k}+\chi_{k}^{*}=4 \gamma\left|\chi_{k}\right|^{2}$ or variations thereof, which is a form of fluctuation-dissipation relation. Although we utilize this identity in our calculation, we observe, however, that this really follows from the dissipative properties of the accelerated detector and its free uncoupled dynamics. It therefore does not explicitly involve the fluctuations of the field. We point out that this cancellation can instead be understood to follow because the correlations between the accelerated detector and probe trajectories can be expressed partly in terms of the noise or field fluctuations along the accelerated trajectory alone, and also because of the isotropy of this noise. The expression of correlation in terms of noise can be equivalently viewed as a consequence of the correlationpropagation relations we obtain in Sec. IV, which are appropriate extensions of a generalized fluctuation-dissipation relation directly relating field fluctuations to dissipative properties.
A distinct feature of the influence functional formalism as used in this paper is the assumption of an uncorrelated fieldoscillator initial state. As argued in the appendix, an uncorrelated initial state is more readily realizable in the derivative coupling model. However, since the minimal and derivative coupling models are dynamically equivalent, we expect our final results to be essentially unchanged, in particular the results of detector response in the various cases studied. The discussion of fluctuation-dissipation relations can be reformulated as well in a way suitable to the derivative coupling model.

We would like to mention possible extensions of this work to other problems. In discussions of the quantum equivalence principle $[29,12]$, one compares the response of a detector moving on a geodesic trajectory in Minkowski space, and coupled to a quantum field, to its response along a geodesic of a spacetime with a homogenous gravitational field. The idea is to derive a suitable transformation on the state of the quantum field which yields the same detector response in both cases. If one can find such a transformation, the equality of the detector response in both cases constitutes a test of the validity of the quantum equivalence principle for local physical processes. However, a homogeneous gravitational field defines a global inertial frame, and so one is inclined to believe that the equivalence principle would hold for nonlocal processes as well, such as the effective dynamics of two spatially separated detectors coupled to the same quantum field. We plan to investigate this and related issues, especially the implications of our findings on black hole back reaction and information problems in later works.

\section{ACKNOWLEDGMENTS}

This work was supported in part by the National Science Foundation under Grant No. PHY94-21849. A.R. would like to thank Professor Ulrich Gerlach for the opportunity to present an early version of this work at the Seventh Marcel Grossmann Meeting in General Relativity, and the University of Maryland Graduate School for partial travel support. B.L.H. acknowledges support from the General Research Board of the Graduate School of the University of Maryland and the Dyson Visiting Professor Fund at the Institute for Advanced Study, Princeton. Part of this work was done while he visited the Newton Institute for Mathematical Sciences at the University of Cambridge during the Geometry and Gravity program. J.A. thanks Salman Habib for discussions, and the Canadian Natural Sciences and Engineering Research Council for support.

\section{APPENDIX A: INFRARED PROBLEMS IN THE MINIMAL COUPLING MODEL}

The infrared effects of the minimal coupling (MC) model are not trivial. In fact, as we will show, the proper treatment of this simple model, including an ultraviolet but not an infrared cutoff, leads us to identify a superselection rule which prefers a particular class of bases in the model's Hilbert space. Using the minimal coupling model and this preferred class of bases is equivalent to a derivative coupling model used by Unruh and Zurek and a basis of direct products of unperturbed field and oscillator states. 
The MC Hamiltonian is

$$
H_{\mathrm{MC}}=H_{\Phi_{0}}+\frac{1}{2 M}[P-\epsilon \phi(0)]^{2}+\frac{\kappa}{2} Q^{2} .
$$

It is straightforward to show that the expectation value of $H_{\mathrm{MC}}$ for soft photon states (i.e., low energy eigenstates of the free field Hamiltonian) has a contribution proportional to the inverse of their unperturbed energy. This suggests that the true low energy states of this model must have strong correlations between the field and the oscillator. If we reject an infrared cutoff as unphysical, then we must conclude that states that are direct products of field and oscillator states will actually have energies very much higher than that of the true ground state of the model.

The poor behavior of the basis of unperturbed (i.e., $\epsilon \rightarrow 0$ ) energy eigenstates reflects the fact that, since there are field modes at all frequencies, expanding around $\epsilon=0$ correctly requires degenerate perturbation theory. Since we are trying to set up an open quantum system, however, our choice of basis is not merely a matter of convention. Different bases can imply different partitions of the complete Hilbert space into "system" and 'environment" subspaces. In the influence functional formalism, one traces over the final states of the environment, and assumes an initial state which is often a direct product of system and environment. If we change what we mean by "system" and "environment," the final trace becomes a different operation, and the initial state becomes a different state.

It is not the model itself, of course, but only the naive basis that is badly behaved: the full Hamiltonian is quadratic, and hence equivalent to a set of decoupled harmonic oscillators. To understand the problems with the basis of unperturbed energy eigenstates, and to identify a better basis, we should diagonalize the full Hamiltonian.

The MC Hamiltonian may be diagonalized by defining new creation and annihilation operators. If the original field and conjugate momentum operators are $\phi(x)$ and $\pi(x)$, the diagonalizing annihilation operators are

$$
\begin{aligned}
a_{k}= & \frac{1}{\sqrt{2 \hbar \pi|k|}}\left(\int_{-\infty}^{\infty} d x\left[C(k) E_{k}(x)+i \sin k x\right]\right. \\
& \times[|k| \phi(x)+i \pi(x)])+\epsilon C(k)[i Q-|k| P],
\end{aligned}
$$

where $\quad E_{k}(x)=\left(1-k^{2} M / \kappa\right) \cos k x-\left[\epsilon^{2} k /(2 \kappa)\right] \sin k|x| \quad$ and $C(k)=\left[\left(1-k^{2} M / \kappa\right)^{2}+\epsilon^{4} k^{2} /\left(4 \kappa^{2}\right)\right]^{-1 / 2}$, for a massless field.

Note that the set of fieldlike operators $\left\{a_{k}, a_{k}^{\dagger}\right\}$ diagonalizes the entire Hamiltonian. There is no normal mode of the coupled system which corresponds even weakly to the unperturbed oscillator, and all of the normal modes contain very nonlocal excitations of the field. If we wish to consider the oscillator as an open system coupled to the field as an unobserved environment, then, the basis of exact energy eigenstates of the combined system will not be particularly convenient. The fact that this model is easily solved exactly does not make the system-environment problem trivial.

We can now, however, determine the effect on the true ground state of the unperturbed oscillator raising and lower- ing operators. We find that either of these operators maps the ground state onto a highly excited state, whose expected energy is infrared divergent. Consequently, observations that are restricted to the oscillator sector alone [as it is defined from Eq. (A1)] may be said to require infinite amounts of energy. Considering the field that appears in Eq. (A1) to be an unobserved environment is therefore unphysical.

We can, however, change our basis so that the Hamiltonian appears more benign in terms of the transformed operators. We may effect this transformation using the unitary operator $U=\exp (-(i / \hbar) \epsilon Q \phi(0))$. This transformation mixes the field and oscillator sectors, and so changes what is meant by an observation of the oscillator alone. We can check that the new oscillator raising and lowering operators, acting on the ground state, now produce states whose energy is ultraviolet divergent, instead of infrared. A physically plausible UV cutoff then renders this energy finite, and it becomes reasonable to consider the new field sector as unobserved. Furthermore, a direct product of the unperturbed ground state of the transformed field and any finite-energy oscillator state now has finite energy, and so is not unreasonable as an initial state for the coupled system.

An alternative way of expressing the advantage of the transformed variables is to say that in order for a degree of freedom in the theory to be observable in isolation, it must require finite energy to excite or de-excite it without affecting other degrees of freedom, and it must also be spatially local (except possibly at small scales). Such an observed degree of freedom will be some linear combination of the exact normal modes. If we require the coefficients in this linear combination to vanish at high energies and remain finite everywhere else, we can ensure finite energy observability. If we require that the coefficients are constant at low energies, we also ensure local observability.

We would then like to have a basis in which this observed linear combination "looks like" a harmonic oscillator coupled to a scalar field. Given our conditions of local and finite energy observability, our original basis does not provide this feature, but our second basis does.

The transformed Hamiltonian $H_{\mathrm{MC}}$ becomes, in the new operators, precisely the Hamiltonian of the Unruh-Zurek model. We have, therefore, found that, even if we begin by analyzing the minimally coupled model, we may be compelled in the end to study the Unruh-Zurek model (with a UV cutoff) instead. More convenient for our subsequent calculations than the Hamiltonian for this model is its Lagrangian:

$$
\begin{aligned}
L_{\mathrm{DC}}= & \frac{1}{2}\left(M \dot{Q}^{2}-\kappa Q^{2}\right)+\frac{1}{2} \int d k \dot{\Phi}_{k}^{*} \dot{\Phi}_{k}-k^{2} \Phi_{k}^{*} \Phi_{k} \\
& -\frac{\epsilon}{\sqrt{2 \pi}} Q\left(\dot{\Phi}_{k}+\dot{\Phi}_{k}^{*}\right) .
\end{aligned}
$$

Here, $\Phi_{k}$ is the time-dependent, spatial Fourier transform of the field $\phi$.

Note that, even in the more benign Unruh-Zurek basis, distinguishing the oscillator as a system observable independently of the field directly implies that there must be a UV cutoff. One often argues that a cutoff is appropriate because one is not interested in accurately describing physics at inaccessible energy scales; but in the case of the oscillator 
coupled to a field, there must really be a cutoff in the coupling in order for there to be any accessible energy scale.

\section{APPENDIX B: CORRESPONDENCE BETWEEN MINIMAL AND DERIVATIVE COUPLING MODELS}

In the minimal coupling model, the derivative of the oscillator coordinate couples to the field, whereas in the derivative coupling model the oscillator coordinate couples to the derivative of the field. These two models thus differ by a total derivative term in the Lagrangian. In particular, they have the same Heisenberg operator dynamics. The above subsection describes the issue of the initial state, and argues that an uncorrelated initial state is physically more realistic in the derivative coupling model. However, since the two models have the same dynamics, this should translate to a simple prescription for switching from one model to the other in the context of the influence functional treatment.

In the previous sections, we have derived all results from the minimal coupling model. One can obtain corresponding quantities in the influence functional of the derivative coupling model via the prescription below:

$$
\begin{gathered}
\frac{d Q_{i}}{d \tau_{i}} \rightarrow Q_{i}, \\
\frac{d Q_{i}^{\prime}}{d \tau_{i}} \rightarrow Q_{i}^{\prime}, \\
Z_{i j}\left(\tau_{i}, \tau_{j}^{\prime}\right) \rightarrow \frac{d}{d \tau_{i}} \frac{d}{d \tau_{j}^{\prime}} Z_{i j}\left(\tau_{i}, \tau_{j}^{\prime}\right) .
\end{gathered}
$$

[1] W.G. Unruh, Phys. Rev. D 14, 870 (1976).

[2] S. Fulling, Phys. Rev. D 7, 2850 (1973).

[3] L. Parker, Phys. Rev. 183, 1057 (1969).

[4] S.W. Hawking, Commun. Math. Phys. 43, 199 (1975).

[5] P.C.W. Davies, J. Phys. A 8, 609 (1975); B.S. DeWitt, Phys. Rep. 19C, 295 (1975).

[6] W.G. Unruh and R.M. Wald, Phys. Rev. D 29, 1047 (1984).

[7] D. Sciama, P. Candelas, and D. Deutsch, Adv. Phys. 30, 327 (1981).

[8] S. Takagi, Prog. Theor. Phys. Suppl. 88, 1 (1986).

[9] V.L. Ginzburg and V.P. Frolov, Sov. Phys. Usp. 30, 1073 (1988).

[10] P.G. Grove, Class. Quantum Grav. 3, 801 (1986).

[11] D.J. Raine, D.W. Sciama, and P. Grove, Proc. R. Soc. London A 435, 205 (1991).

[12] W.G. Unruh, Phys. Rev. D 46, 3271 (1992).

[13] S. Massar, R. Parentani, and R. Brout, Class. Quantum Grav. 10, 385 (1993).

[14] F. Hinterleitner, Ann. Phys. (N.Y.) 226, 165 (1993).

[15] J. Audretsch and R. Müller, Phys. Rev. D 49, 6566 (1994).

[16] B.L. Hu, "Quantum Statistical Fields in Gravitation and Cosmology," in Proc. Third International Workshop on Thermal Field Theory and Applications, edited by R. Kobes and G. Kunstatter (World Scientific, Singapore, 1994), Report No. grqc/9403061 (unpublished).

[17] B.L. Hu, Physica A 158, 399 (1989).
The stochastic effective action in the derivative coupling model is then given by

$$
\begin{aligned}
S_{\mathrm{eff}}= & \sum_{i=1}^{N} \int_{-\infty}^{t_{i}^{-1}(T)} d \tau_{i}\left[\dot{Q}_{i}^{-} \dot{Q}_{i}^{+}-\Omega_{i}^{2} Q_{i}^{-} Q_{i}^{+}-Q_{i}^{-} s_{i}\left(\tau_{i}\right) \eta_{i}\left(\tau_{i}\right)\right. \\
& -2 Q_{i}^{-} s_{i}\left(\tau_{i}\right) \sum_{j=1}^{N} \int_{-\infty}^{t_{j}^{-1}\left(t_{i}\left(\tau_{i}\right)\right)} d \tau_{j}^{\prime} Q_{j^{\prime}}^{+} s_{j}\left(\tau_{j}^{\prime}\right) \tilde{\mu}_{i j}\left(\tau_{i}, \tau_{j}^{\prime}\right) .
\end{aligned}
$$

Note that the quantities $\widetilde{\mu}_{i j}$ in the above equation refer to the newly defined quantities in the derivative coupling model. They are obtained by differentiating the corresponding quantities in the MC model twice.

The Langevin equations are

$$
\begin{aligned}
& \frac{d^{2} Q_{i}}{d \tau_{i}^{2}}-2 \sum_{j=1}^{N} \int_{-\infty}^{t_{j}^{-1}\left(t_{i}\left(\tau_{i}\right)\right)} d \tau_{j}^{\prime} s_{j}\left(\tau_{j}^{\prime}\right) s_{i}\left(\tau_{i}\right) \tilde{\mu}_{i j}\left(\tau_{i}, \tau_{j}^{\prime}\right) Q_{j}+\Omega_{i}^{2} Q_{i} \\
& \quad=s_{i}\left(\tau_{i}\right) \eta_{i}\left(\tau_{i}\right) .
\end{aligned}
$$

The noise kernel, as the correlator of $\eta_{i}$ and $\eta_{j}$, is also obtained by the corresponding noise kernel in the MC model by differentiating twice, according to the correspondence established above.

The infrared-divergent energy of the initially uncorrelated state does have an effect: the propagation kernel, in the minimal coupling model, contains an initial "shock wave", term, as well as the expected dissipation and propagation terms, and this term is not present in the derivative coupling model. Since this shock wave is a transient, it has no significance in our late-time analysis.
[18] B.L. Hu, in Proceedings of the CAP-NSERC Summer Institute in Theoretical Physics, Vol. 2, Edmonton, Canada, 1987, edited by K. Khanna, G. Kunstatter, and H. Umezawa (World Scientific, Singapore, 1988).

[19] E. Calzetta and B.L. Hu, Phys. Rev. D 37, 2878 (1988).

[20] E. Calzetta and B.L. Hu, "Decoherence of Correlation Histories," in Directions in General Relativity, Vol. II: Brill Festschrift, edited by B.L. Hu and T.A. Jacobson (Cambridge University Press, Cambridge, England, 1993).

[21] E. Calzetta and B.L. Hu, "Correlations, Decoherence, Disspation and Noise in Quantum Field Theory,' in Heat Kernel Techniques and Quantum Gravity, edited by S. Fulling (Texas A\&M University Press, College Station, TX, 1995).

[22] B.L. Hu, J.P. Paz, and Y. Zhang, Phys. Rev. D 45, 2843 (1992); 47, 1576 (1993).

[23] B.L. Hu and A. Matacz, Phys. Rev. D 49, 6612 (1994).

[24] E. Calzetta and B.L. Hu, Phys. Rev. D 49, 6636 (1994).

[25] J.R. Anglin, Phys. Rev. D 47, 4525 (1993).

[26] D.W. Sciama, "Thermal and Quantum Fluctuations in Special and General Relativity: an Einstein Synthesis," in Centenario di Einstein (Editrici Giunti Barbera Universitaria, 1979).

[27] I.S. Gradshteyn and I.M. Ryzhik, Table of Integrals, Series, and Products (Academic, New York, 1965), p. 684 (6.561-16) and p. 937 (8.332-3).

[28] I.S. Gradshteyn and I.M. Ryzhik [27], p. 503 (3.981-1).

[29] P. Candelas and D.W. Sciama, Phys. Rev. D 27, 1715 (1983).

[30] R.P. Feynman and F.L. Vernon, Ann. Phys. (N.Y.) 24, 118 (1963). 